\title{
Role of Planetary Boundary Layer Processes in the Simulation of Tropical Cyclones Over the Bay of Bengal
}

\author{
K. Vijaya Kumari, ${ }^{1}$ (D) S. Karuna Sagar, ${ }^{1,4}$ V. Yesubabu, ${ }^{2}$ D. Hari Prasad, ${ }^{3}$ and S. Vijaya Bhaskara Rao ${ }^{1}$
}

Abstract-The behaviour of planetary boundary layer (PBL) schemes initialized at different life stages of a tropical cyclone (TC) is studied by considering seven Bay of Bengal TC cases. In each TC case, the Advanced Research Weather Research and Forecasting (WRF-ARW) model is initialized at four life stages (depression to very severe cyclone storm) with National Center for Environmental Prediction (NCEP) Global analysis and integrated up to $96 \mathrm{~h}$. A set of six PBL sensitivity experiments are conducted at four stages for all seven TC cases to analyse the impact of the model boundary layer in simulating the TC track and intensity parameters. The model-produced track, intensity and rainfall patterns are evaluated with the best track, intensity and gridded rainfall estimates obtained from the India Meteorological Department (IMD). The spatial and radius/height section simulated fields are evaluated with satellite retrievals. Results depict that the six PBL schemes during model initialization at different stages of a TC have produced sizable differences in the simulation of track and intensity parameters. The local and nonlocal schemes produced different results based on the TC stage at which the model is initialized. The results also suggest that if the model is initialized with a non-organized cyclonic vortex such as depression stage of the storm, PBL schemes exhibit high sensitivity and spread in terms of both track and intensity. While the spread between PBL schemes was significantly reduced and found close to the observed estimates when the model was initialized at the advanced stages of the TC. In addition, the local 1.5-order closure scheme simulated the storm parameters relatively better when the cyclone vortex was not well organized in the model's initial conditions, while the non-local and first-order closure schemes perform better with initial model conditions of a well-defined cyclonic vortex.

1 Department of Physics, S. V. University, Tirupati 517502, India.

2 Department of Space (DoS), National Atmospheric Research Laboratories (NARL), Govt. of India, Gadanki, Chittoor District, Tirupati, Andhra Pradesh 517 112, India. E-mail: yesubabu@narl.gov.in

3 Physical Sciences and Engineering Division, King Abudullah University of Science and Technology (KAUST), Thuwal 23955-6900, Kingdom of Saudi Arabia.

4 National Centre for Medium Range Weather Forecasting (NCMRWF), Ministry of Earth Sciences (MoES), Noida 201309 , India.
Key words: Tropical cyclones, WRF model, planetary boundary layer, post monsoon, Bay of Bengal.

\section{Introduction}

The Indian subcontinent receives most of the rainfall during the southwest monsoon and the amount of annual rainfall varies with the periodical formation of synoptic disturbances over the Bay of Bengal (BOB) and Arabian Sea (Samala et al. 2013). Rain shadow regions located in the southern part of India, like Tamil Nadu and south-western parts of Andhra Pradesh and Orissa, receive a significant portion of rainfall during the post-Monsoon season (October-December) due to the formation of depressions and tropical cyclones (TCs). The annual rainfall over these regions highly depends on the depressions and TCs (Kumar et al. 2010) formed under the favourable atmospheric thermo-dynamical conditions (Gray 1968) over the BOB. Though TCs play a critical role in transferring heat and moisture from the regions of warmer lower latitudes to colder higher latitudes, they can cause extensive damage to lives as well as property, especially in coastal regions. Therefore, improvements in an advanced accurate prediction of TCs in terms of intensity, track and associated wind along with its rainfall is essential to give lead time forecast warnings and to mitigate the disasters, especially over coastal areas to avoid loss of life and property. Conventional studies indicate the prediction of the cyclone movement is challenging over the BOB due to its higher variability (Raghavan and Sen Sarma 2000). However, recent studies reveal that the capability of cyclone track prediction is improved significantly with the supply

\begin{tabular}{|l|lll|}
\hline & Journal : Small 24 & Dispatch : 11-10-2018 & Pages : 27 \\
Article No. : 2017 & $\square$ LE & $\square$ TYPESET \\
& MS Code : PAAG-D-18-00007 & $\checkmark \mathrm{CP}$ & $\checkmark$ DISK \\
\hline
\end{tabular}


of realistic initial conditions, though the prediction of cyclone intensity still needs to be improved (Mohanty et al. 2014; Yesubabu et al. 2014a; Mandal et al. 2016).

Simulation of TCs using a numerical weather perdition model is highly sensitive to several factors such as initial conditions, horizontal and vertical model grid resolutions (Bhaskar Rao et al. 2009; 2010) and physical parameterization schemes, etc. (Ma et al. 2012; McFarquhar et al. 2006; Fovell and Su 2007). Many studies (Mandal et al. 2004; Bhaskar Rao and Hari Prasad 2007; Pattnaik and Krishnamurti 2007; Srinivas et al. 2012) have been carried out to examine the numerical model sensitivity to various physics parameterization schemes in regards to both individual impact and combined impact in predicting the facets of TCs. Out of all model physics schemes, the planetary boundary layer (PBL) plays a vital role for TC predictions over any basin (Anthes 1982). The PBL turbulence plays an influential role in the simulation of low-level circulation and the alteration of primary and secondary circulation of a TC, genesis of clouds and diffusion of dynamical and thermo-dynamical quantities, which are highly influential in simulation of a TC's track, intensity, structure, and also the TC-induced rainfall (Kepert 2012). Also, the boundary layer physics plays a critical role in modulating the energy transfer from the ocean surface to the atmosphere which occurs through the ocean mixed layer (Braun and Tao 2000). A number of studies (Langousis et al. 2009; Smith et al. 2009, 2014; Smith and Thomsen 2010) have shown that the surface drag and boundary layer diffusion play a crucial role in the transport of heat, moisture and momentum transport, and thus influence the lowlevel winds, frictional convergence, structure and intensification of the cyclone. Mellor and Yamada (1982) showed that the use of higher-order PBL schemes based on prognostic turbulent kinetic energy (TKE) closure gives considerably higher intensity estimates than the first-order, non-local schemes based on eddy diffusivity approaches such as the Yonsei University Scheme (YSU; Hong et al. 2006).

In the North Indian Ocean (NIO), several studies have reported the advantages of high-resolution mesoscale models for forecasting TCs using the Fifth-Generation Penn State/NCAR Mesoscale Model
(MM5) and Weather Research and Forecasting (WRF) models (Mohanty et al. 2004; Bhaskar Rao and Hari Prasad 2006; Bhaskara Rao et al. 2007, 2009, 2010; Srinivas et al. 2007; Srikanth et al. 2016; among others). Most of these studies are restricted to a single TC case study to understand the model's sensitivity with different PBL physics (e.g., Mandal et al. 2004; Srinivas et al. 2007, 2013; Deshpande et al. 2010; Ancy et al. 2014). Srinivas et al. (2007) studied the impact of convection, PBL and explicit moisture process on the track and intensity prediction of the Andhra severe cyclone (2003) using MM5. They showed that the Mellor-Yamada TKE closure simulated a highly intense storm and the k-profilebased non-local diffusion scheme, medium-range forecast (Hong and Pan 1996) produced the best simulation in terms of track and intensity. Osuri et al. (2014) studied the impact of surface roughness on the intensity of a TC Aila using the WRF model. They reported that the role of roughness length is less impactful on the simulation TC intensity during the developing stage; when the TC approaches the coast, higher values of surface roughness length produced relatively better performance in simulation of the TC's track and intensity.

Apart from the sensitivity studies of individual TC cases, there are also studies such as Srinivas et al. (2012) in evaluating the performance of the WRF model for five severe BOB cyclones by varying the convection, microphysics, boundary layer and land surface parameterisation schemes. Substantial changes in TC intensity are reported with the variation of PBL schemes and it is concluded that the physics combination of the Kain-Fritsch convection scheme (KF), Lin microphysics scheme, YSU PBL and NOAH Land Surface Model (NOAH-LSM) options provide the least errors for operational TC forecasting. Osuri et al. (2012) also showed similar results while evaluating the performance of different *parameterization schemes for five TCs formed over the NIO. Recently, Sateesh et al. (2017) showed that the YSU PBL scheme produced better results in terms of wind, pressure distribution and rainfall compared to the Mellor-Yamada-Janjic (MYJ) scheme in the simulation of TC Thane over the BOB with the model initialized at the cyclone stage of the TC. Chandrasekar and Balaji (2012) pointed out the model

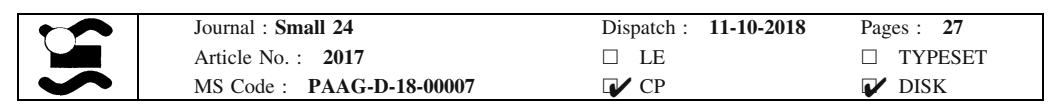


sensitivity in simulation of TCs highly depends on the prevailing synoptic conditions. The modelling studies have also looked at the impact of initial conditions over BOB cyclones (Mohanty et al. 2010; Chandrasekar and Balaji 2016; Yesubabu et al. 2014b). Srinivas et al. (2013) analysed the significance of the model initialising at different stages of the cyclone and the results highlight the better performance of the model initialized at a mature stage of the cyclones. Greeshma et al. (2015) also reported the consistent improvement in the model predictions when the TCs are initialised at depression and mature stages, and the increased skill of the model during mature stages is attributed to the precise representation of the vortex of the cyclones in the model through the initial conditions.

In this study, we have evaluated the role of PBL schemes in simulating TCs by initializing the WRF model at different TC life stages. The PBL sensitivity of the model is verified using the initializations made at four TC life stages based on the intensification, i.e., depression (DEP), deep DEP (DD), cyclonic storm (CS) and very severe cyclonic storm (VSCS). We have chosen the TC cases formed during the post-monsoon season as the majority of CSs formed over the BOB are during this season. In addition, to maintain similar mean synoptic conditions for all the TC cases, we have considered the TCs which moved in a north to north-westerly direction. Based on the above criteria, we have considered seven TC cases and evaluated the skill of six PBL schemes initialized at four TC life stages. Further, we have made detailed track and intensity analysis of the best-performing TC case. This paper is organized as follows: Sect. 2 provides brief notes on the PBL schemes, Sect. 3 details the WRF model and physics configuration, the results are provided in Sect. 4, and finally, summary and conclusions are given in Sect. 5.

\section{Description for PBL Schemes}

The PBL turbulence has an influential role in the atmospheric dynamics and simulation of TCs due to vertical mixing which can be parameterized more precisely based on the quantities resolved with the mesoscale model. The spin of the cyclone vortex has an association with the dynamics of the PBL (Montgomery et al. 2010; Smith et al. 2014), suggesting the importance of the boundary layer in numerical models. The details of six PBL schemes used in this study in the simulation of TCs formed over the BOB during the post-monsoon are presented below.

\subsection{Yonsei University (YSU) Scheme}

The YSU scheme, as a first-order closure scheme, is a revised algorithm of general circulation models as well as medium-range forecasting models (Holtslag and Boville 1993; Hong and Pan 1996). This model incorporates the entrainment process at the top of the PBL which enhances the mixing during thermal free convection and decreases during the mechanically forced convection.

\subsection{Mellor-Yamada-Janjic (MYJ) Scheme}

The MYJ scheme is developed based on Mellor and Yamada (1982) and is a local vertical mixing one-dimensional prognostic TKE PBL scheme with orders of 1.5 and level 2.5. It includes a term along with the heat and moisture fluxes that control them against their local gradients. This effect has been combined along with TKE and leads to the complete representation of the entrainment.

\subsection{Mellor-Yamada Nakanishi-Niino (MYNN) Scheme}

The closure constants in the Mellor and Yamada turbulence closure model were re-evaluated and a new prognostic equation for master scale length has been proposed in the MYNN model. It includes the liquid water potential temperature, total water content and condensation (Nakanishi and Niino 2004) in a model grid in order to interact with microphysics and radiation. In addition, the improved MYNN model can reproduce the Kelvin-Helmholtz instability and periodic oscillations which was not possible in the previous version of the MYNN model.

\begin{tabular}{|l|lll|}
\hline & Journal : Small 24 & Dispatch : 11-10-2018 & Pages : 27 \\
& Article No. : 2017 & $\square$ LE & $\square$ TYPESET \\
& MS Code : PAAG-D-18-00007 & $\downarrow \mathrm{CP}$ & $\checkmark$ DISK \\
\hline
\end{tabular}




\subsection{Asymmetric Convective Model (ACM) Version 2 Scheme}

The Asymmetric Convective Model version 1 (ACM1) is a modified version of the Blackadar convective model (Blackadar 1978). The convective transport initialized in the surface layer of the model can spread all other layers in the PBL. The major drawback of ACM1 is that there is no representation of local diffusion in the upward direction which results in unrealistic transport from the lower layer to the upper layers. This drawback has been resolved in ACM version 2 (ACM2) by introducing the eddy diffusion component which can simulate the vertical profiles more accurately even when the gradient is decreasing near the surface layer.

\subsection{Bougeault-Lacarrere (BouLac) Scheme}

The Bougeault-Lacarrere scheme, mainly designed to include the orographically induced turbulence, is a 1.5-order local closure scheme widely used in air quality applications. The option of TKE prediction is included by introducing an additional prognostic equation. Once the TKE intensity was predicted, eddy diffusion coefficients and mixing lengths will be well defined for improved simulations of dynamic fields (Bougeault and Lacarrere 1989).

\subsection{Grenier-Bretherton-McCaa (GBM) Scheme}

This scheme is also a 1.5-order local closure that includes TKE prognostic equation, designed to study the stratocumulus-capped boundary layers (Grenier and Bretherton 2001). The scheme employs moist thermodynamics based on the formulation proposed by Mellor and Yamada (1982). The vertical transport of TKE is increased for proper comparison with the large eddy simulations. The scheme performs well in dry convection cases and was tested for both fine as well as coarse vertical resolutions in a single column model.
3. Data, Model Configuration and Experimental Details

Advanced Research WRF (WRF-ARW) version 3.8.1 is used in the present study in the simulation of a TC formed over the BOB. The terrain data for two domains used for this study is presented in Fig. 1a. The outer domain with $27 \mathrm{~km}$ includes Asian land and NIO and the inner domain with $9 \mathrm{~km}$ covers the $\mathrm{BOB}$ and Indian land regions. Details of model and parameterization schemes used for the study are given in Table 1. The model simulations are carried out with the best combination of microphysics, land surface, radiation and cumulus parameterization schemes adopted from the previous studies (Srinivas et al. 2012; Osuri et al. 2013; Kanase et al. 2015; Singh and Bhaskaran 2017). The WRF model is initialized with the National Center for Environmental Prediction Global Forecast System (NCEP-GFS) Analysis $0.5^{\circ} \times 0.5^{\circ}$ resolution data, integrated up to $96 \mathrm{~h}$, and boundaries are updated at 6-h intervals. A suite of approximately 168 simulations was carried out for seven cyclones with six combinations of PBL schemes at four stages of TC initialization. The comparison and performance analysis of the results include a comparison of track positions, maximum sustained winds (MSW), central sea level pressure (CSLP), gradient wind, temperature anomaly, and winds with the observational data.

Track positions, CSLP and MSW are considered from the TC best track data of IMD. Cooperative Institute for Research on Atmosphere (CIRA) products of multi-satellite wind analysis, radius of maximum winds (RMW) and radius-height cross sections of temperature anomaly are used for the model comparison. The accuracy of the multi-satellite wind analysis is described in Knaff and DeMaria (2006) and Knaff et al. (2007) based on the verification analysis of radiosondes carried out during 2007. The quantitative error analysis with different radiosonde data sets indicates that the bias and mean absolute error (MAE) for the estimates of maximum wind radii in all the storm quadrants were found be around 3 and 15 knots, respectively. The evaluation of TC radius and height estimates for gradient wind and a temperature anomaly has been carried out by Demuth et al. (2004) and their results indicate that the

\begin{tabular}{|l|lll|}
\hline & Journal : Small 24 & Dispatch : 11-10-2018 & Pages : 27 \\
& Article No. : 2017 & $\square \mathrm{LE}$ & $\square$ TYPESET \\
MS Code : PAAG-D-18-00007 & $\square \mathrm{CP}$ & $\checkmark$ DISK \\
\hline
\end{tabular}


(a)

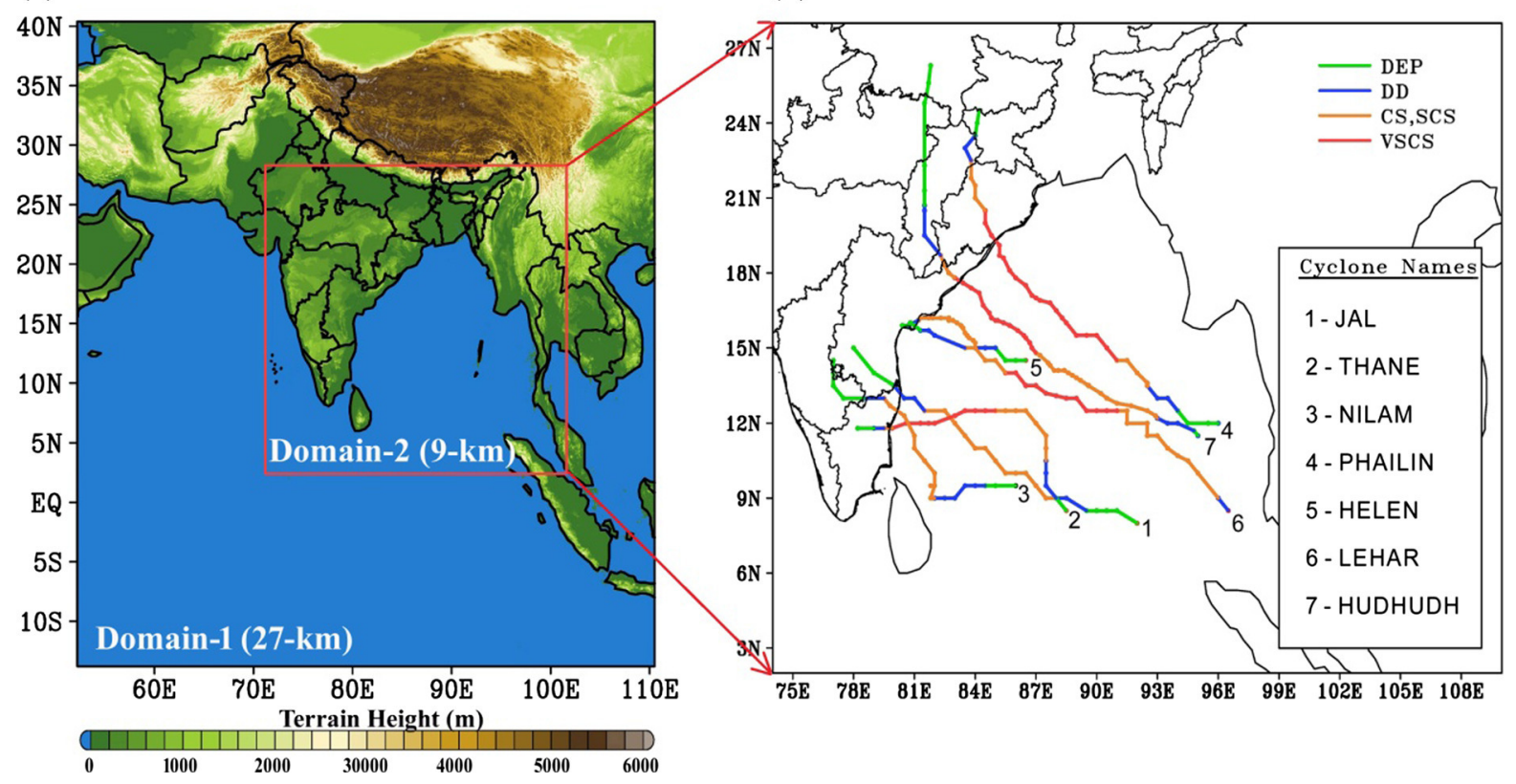

Figure 1

a Two-way nested WRF model domains used in the study along with terrain height. b IMD best track positions of seven cyclone cases considered

Table 1

Model configuration and parameterization schemes

\begin{tabular}{|c|c|}
\hline No. of domains & 2 \\
\hline $\begin{array}{l}\text { Inner domain } \\
\text { dimensions }\end{array}$ & $71.2^{\circ} \mathrm{E}-101.6^{\circ} \mathrm{E} ; 2.4^{\circ} \mathrm{N}-28.3^{\circ} \mathrm{N}$ \\
\hline $\begin{array}{l}\text { Horizontal grid } \\
\text { resolution }\end{array}$ & $27 \mathrm{~km}, 9 \mathrm{~km}$ \\
\hline Vertical layers & 51 \\
\hline Micro physics & Lin et al. scheme \\
\hline Cumulus scheme & KF scheme \\
\hline Land surface physics & NOAH LSM \\
\hline $\begin{array}{l}\text { Radiation scheme for } \\
\text { shortwave }\end{array}$ & $\begin{array}{l}\text { Rapid radiative transfer model for } \\
\text { global (RRTMG) }\end{array}$ \\
\hline $\begin{array}{l}\text { Radiation scheme for } \\
\text { longwave }\end{array}$ & $\begin{array}{l}\text { Rapid radiative transfer model for } \\
\text { global (RRTMG) }\end{array}$ \\
\hline \multirow[t]{6}{*}{ PBL schemes } & YSU \\
\hline & MYJ \\
\hline & MYNN \\
\hline & $\mathrm{ACM}$ \\
\hline & GBM \\
\hline & BouLac \\
\hline
\end{tabular}

MAE and RMSE for gradient winds are found to be in the range of 10.7-11.0 and 14.1-14.9 knots, respectively, and the linear regression analysis reveals that the AMSU-derived radius-height temperature anomaly shows temperature deviations in the range of $0.5-3.5^{\circ} \mathrm{C}$. The wind observations used in the validation of PBL schemes are qualitycontrolled radiosonde data obtained from IGRA and the errors in the wind field are very small $(0.5<\mathrm{m} / \mathrm{s})$ and clearly described in Durre et al. (2006). The rainfall verification analysis is carried out for the model predictions with all the PBL schemes by using the well-known contingency table, i.e., yes/no forecasts (WMO 2008). Various thresholds for the rainfall categories are assigned by following the reports of the India Meteorological Department (IMD 2014). To evaluate the rainfall prediction skill of the model, we computed three widely used skill scores: threat score (TS), percent correction (PC) and false alarm ratio (FAR; Murphy 1993). The 24-h simulated rainfall is compared with the IMD observations, satellite-derived rainfall data sets from Tropical Rainfall Measuring Mission (TRMM) and CPC MORPHing technique (CMORPH) available at a horizontal grid resolution of $0.25^{\circ} \times 0.2$. The performance evaluation analysis of multi-satellite precipitation estimates by Sunilkumar et al. (2015)

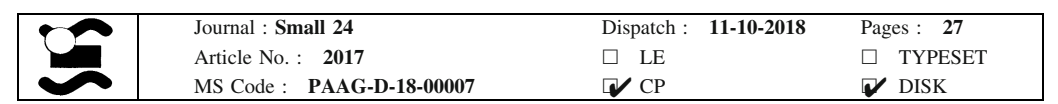


359 revealed that the magnitude of bias (27\%), FAR $360(17.7 \%)$ and RMSE $13.9(\mathrm{~mm})$ are less, and POD $361(62 \%)$ is high for the CMORPH during the north-east 362 monsoon seasons (from 2000 to 2010). Their study 363 also indicates the CMORPH and TRMM display 364 good performance in detecting low to moderate rain 365 events out of other precipitation estimates.

\subsection{Short Description of the Life Cycle of Selected Seven Cyclones}

As described in the introduction, we have selected BOB cyclones propagated in north-west direction during the post-monsoon season (Fig. 1b). More details, such as duration of the cyclone, landfall and its category, etc., are given in Table 2. The Jal TC is a severe cyclonic storm developed over the BOB and crossed as a DD near the north of Chennai on 07 November 2010; it produced a good amount of rainfall over the southern part of Andhra Pradesh. The Thane TC is a VSCS, formed over the BOB as a DEP on 25 December 2011 and crossed Pondicherry on 30 December 2011. The Nilam TC formed on 28 October 2012 as a DEP over the BOB and moved with many changes in directions and made landfall near Mahabalipuram on 31 October 2012. VSCS Phailin developed over the BOB with minimum central sea level pressure of $940 \mathrm{hPa}$ and maximum sustained wind speed of $\sim 60 \mathrm{~ms}^{-1}$ crossed at Gopalpur, Orissa on 12 October 2012. Helen and Lehar cyclonic storms formed one after the other and moved in northwest directions. Helen originated over the $\mathrm{BOB}$, whereas Lehar is a remnant cyclonic circulation from South China Sea. Both the cyclones made landfall over Machilipatnam, Andhra Pradesh
(22 November-Helen, 28 November-Lehar). VSCS Hudhudh formed over the BOB as a lowpressure system on 06 October 2014 and crossed at Visakhapatnam, Andhra Pradesh, on 12 October 2014.

\section{Results and Discussion}

This section is organized in two sub-sections, the first sub-section discusses the model performance based on the initialization at different stages of seven TCs and the second sub-section is the analyses of the results of PBL sensitivity for the TC Phailin case with different model initialization times. The results presented and discussed in the following sections are obtained from a high-resolution model domain (9 km).

\subsection{Quantitative Errors for TC Track and Intensity}

The mean error (ME) and standard deviation (SD) along with its thresholds of ME + SD and ME - SD are computed for the seven TCs in term of the vector track position, CSLP and MSW to illustrate the response of initial model conditions at different stages of the cyclone to different boundary layer schemes. The ME track error (Fig. 2) for seven TCs is in the order of $30-50,100-120,150-90$ and $200-250 \mathrm{~km}$ at $24,48,72$ and $96 \mathrm{~h}$, respectively, indicating the magnitude of the error is increased with the forecast lead time. Considerable reduction of the track error is noticed when the model is initialized at the DD stage rather than the DEP stage. The SD of the PBL schemes is large when the forecast length is

Table 2

Selected cyclones for the study formed in Bay of Bengal and moved in North west ward direction from 2010 to 2014

\begin{tabular}{llll}
\hline No. & Name & Duration & Landfall \\
\hline 1 & Jal & $04-07$ November 2010 & Chennai \\
2 & Thane & $27-30$ December 2011 & PCS \\
3 & Nilam & 28 October-01 November 2012 & Mahabalipuram \\
4 & Phailin & $08-14$ October 2013 & Gopalpur, Orissa \\
5 & Helen & $19-23$ November 2013 & Machilipatnam, AP \\
6 & Lehar & 23-28 November 2013 & Machilipatnam, AP \\
7 & Hudhudh & $07-14$ October 2014 & Visakhapatnam, AP \\
\hline
\end{tabular}

CS cyclonic storm, SCS severe cyclonic storm, VSCS very severe cyclonic storm

\begin{tabular}{|l|lll|}
\hline & Journal : Small 24 & Dispatch : 11-10-2018 & Pages : 27 \\
& Article No. : 2017 & $\square \mathrm{LE}$ & $\square$ TYPESET \\
MS Code : PAAG-D-18-00007 & $\square \mathrm{CP}$ & $\checkmark$ DISK \\
\hline
\end{tabular}


(a)

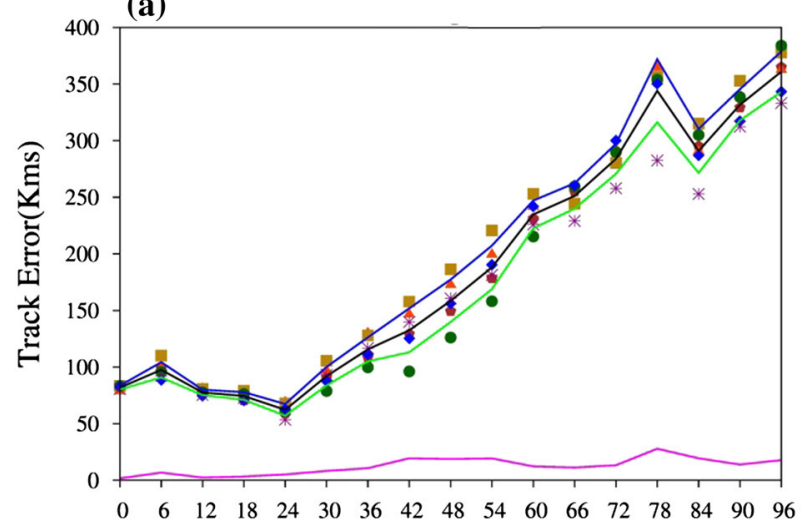

(c)

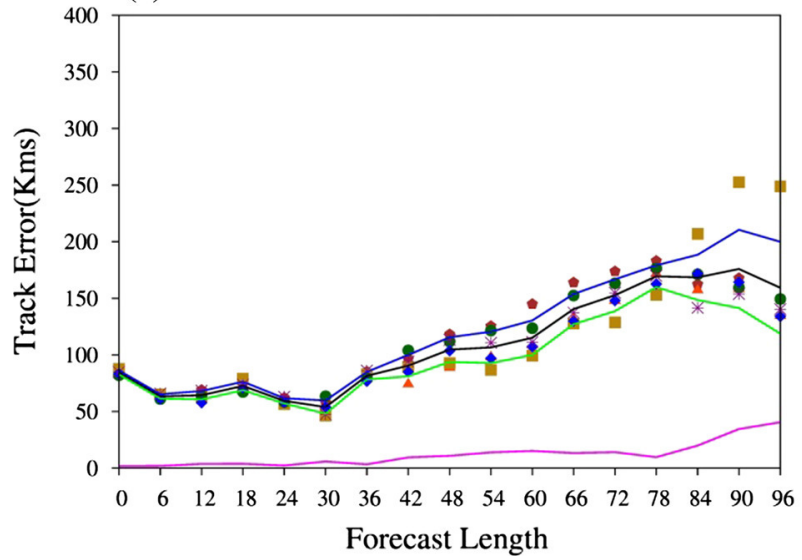

(b)

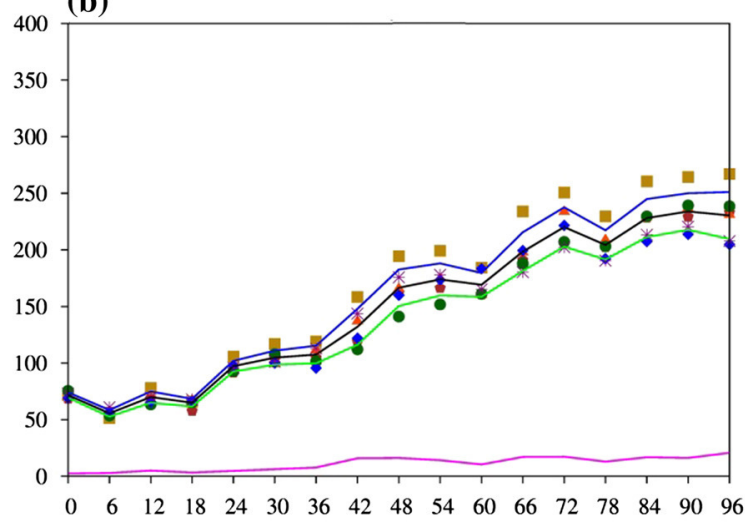

(d)

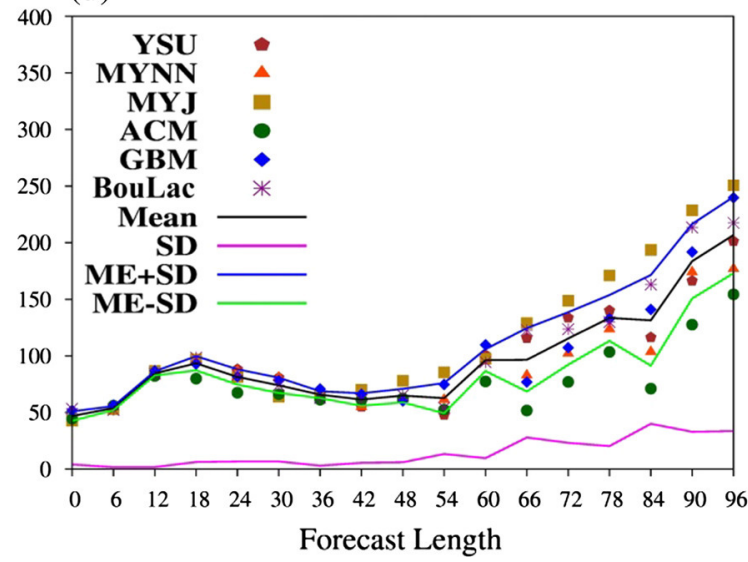

Figure 2

Mean errors in the vector track position computed for all seven cyclones with the model initializations at four stages of TC: a DEP, b DD, c CS and d VSCS

increased, and the error and deviation from its mean is negligible when the model is initialized at DEP stage of the cyclone. This could be due to the welldefined cyclone vortex at the mature stage of the system in these experiments. During the DEP to CS stages, model storm location is dislocated around $30-60-\mathrm{km}$ from the observed storm vortex, and may have enhanced the track errors. These initial errors are mainly due to the interpolation of global analysis and lack of assimilation at the time of mesoscale model initialization. Even though the mean model track errors are increasing with the forecast lead times, the sensitivity to the PBL in terms of local and non-local schemes remains the same. The schemes selected for this study are 1.5-order closure and local schemes, except for YSU and ACM schemes which are first-order non-local schemes. From ME analysis, it is observed that the local schemes performed better when the cyclone vortex is defined more accurately upon model initialization and the model exhibited higher errors when it is initialized at the TC developing stage. The response of the non-local schemes in simulating the position of the cyclone is quite good when the system is well organized, i.e., before and during the mature stage. From the sensitivity of the PBL schemes, the track error is minimal for the non-local schemes (i.e., YSU and ACM), whereas with the local schemes (i.e., MYJ, BouLac, GBM and MYNN), the track error is higher when the model is initialized at DEP, DD and VSCS stages. It infers that the PBL schemes exhibit high sensitivity with improper cyclone vortex at the time of model initialization. This can be noticed from the non-local and $1^{\text {st }}$-order closure schemes simulated the storm positions accurately when the cyclone vortex is not well established at time of model initialization

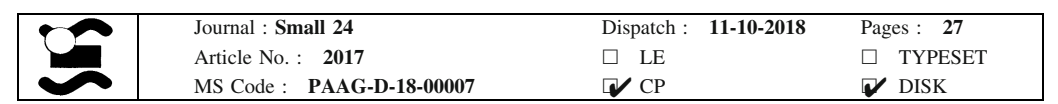


458 and the local 1.5-order closure scheme perform better 459 if we initialize the mesoscale model in the advanced 460 stage of the cyclonic storm. Also, reduction of the 461 initial error from DEP to mature stages suggests the 462 importance of the precise representation of the 463 cyclonic vortex in the initial model conditions.

464 The spatial plot of simulated vector tracks for the 465 seven selected cyclones initialized at four stages of with best track estimates of IMD are shown in Fig. 3.

The comparison of simulated tracks suggests that the deviation from the observed track positions is increasing for all the schemes with the lead time. With model initialization at the DEP stage, the spread between the tracks with PBL schemes is high and it starts reducing with initialization at DD stage; also, the faster movement of the storm is reduced as compared to DEP, except in the case of Hudhudh. At CS/VSCS stage initialization, the deviations in simulated tracks and the movement of the storm are reduced significantly for all seven cyclone cases. Thus, the spread between the tracks simulated by
469

470

471

472

473

474

475

476

477

478

479
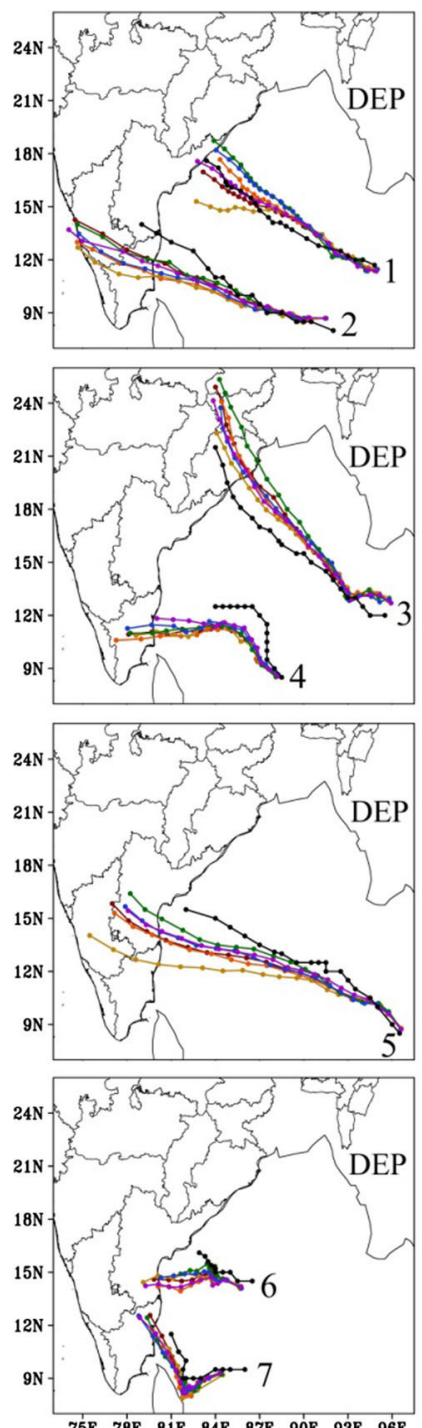
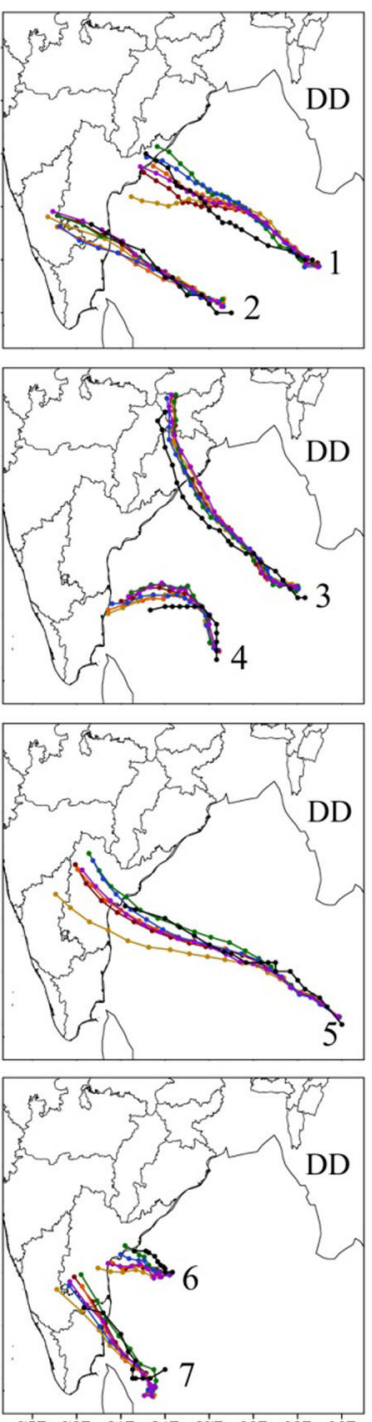
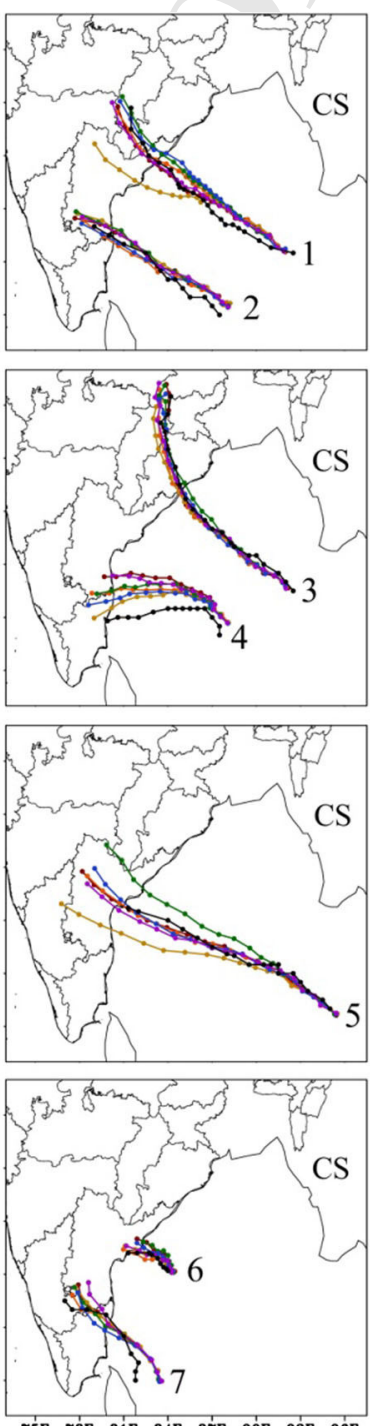
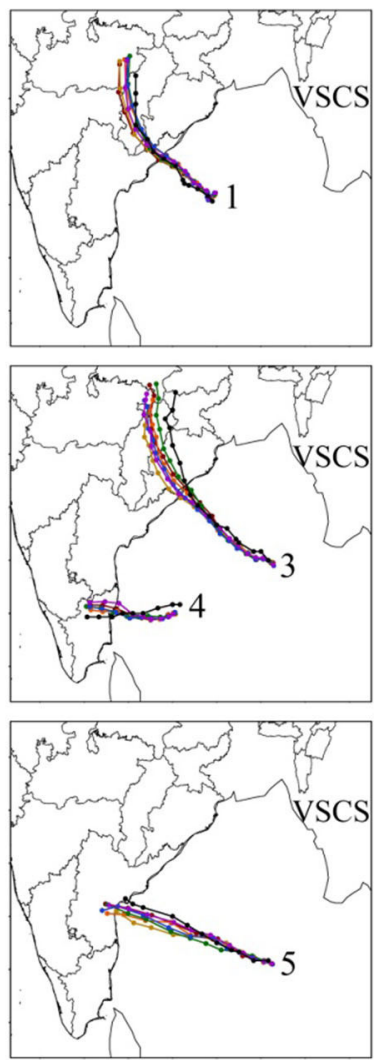

1. Hudhudh IMD -

2. Jal YSU -

3. Phailin MYNN 4. Thane MYJ5.Lehar $\quad$ ACM 6.Helen 7. Nilam BouLac -

Figure 3

Model-simulated vector tracks for all selected cyclones with PBL schemes initialized at four cyclone stages

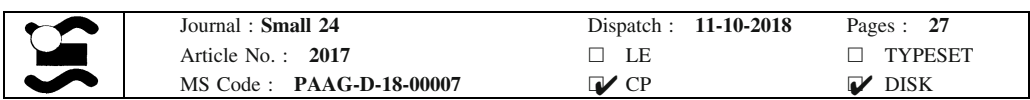


480 different PBL schemes is relatively high at the TC 481 developing phase, while the spread is decreasing as 482 we move model initialization time to cyclonic and 483 VSCS, suggesting that the sensitivity of PBL is large 484 when the model initializes in the DEP stage rather 485 than in other initialization times. Out of all the PBL 486 schemes, the MYJ scheme followed by the ACM 487 scheme showed higher deviations from the observed 488 track estimates, and the BouLac PBL physics 489 produced the best results for the TC track simulation 490 followed by the YSU scheme. TC errors in CSLP (Fig. 4), MSW (Fig. 5) between the simulated values (WRF) and IMD estimates for each PBL schemes averaged for the seven TC cases.
The ME and SD errors in CSLP are in the order of -6.6 to $-1,-12.5$ to -2.9 and -14.3 to $-0.1 \mathrm{hPa}$ and $1.18,3.18$ and $4.4 \mathrm{hPa}$ for $24-$, 48- and 72-h simulations, respectively. Meanwhile, in the case of MSW, the ME and SD errors are between 2.7 to 5.6, 1.7 to 6.7 and -3.4 to $3.4 \mathrm{~ms}^{-1}$ and $1.2,1.6$ and $2.1 \mathrm{~ms}^{-1}$ at 24,48 and $72 \mathrm{~h}$, respectively. It is seen that the model overestimates the cyclone intensity both in terms of CSLP and MSW with all PBL schemes and the intensity errors are found to be large between 42 and $72 \mathrm{~h}$ of model integration which is during the mature stage of the considered TC cases. The ME for CSLP (Fig. 4) is negative and MSW is positive during the deepening phase of the simulated storm when we initialize the model in DEP, DD and CS stages for all the PBL schemes; this suggests that

\section{(a)}

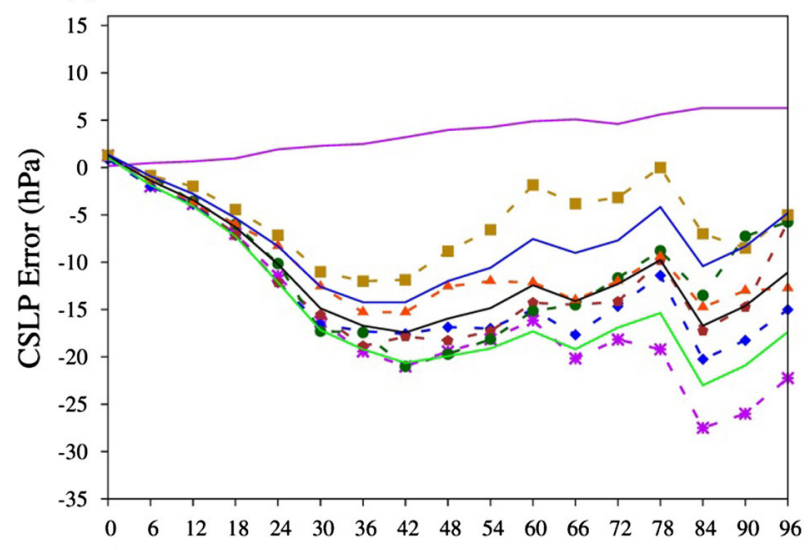

(b)

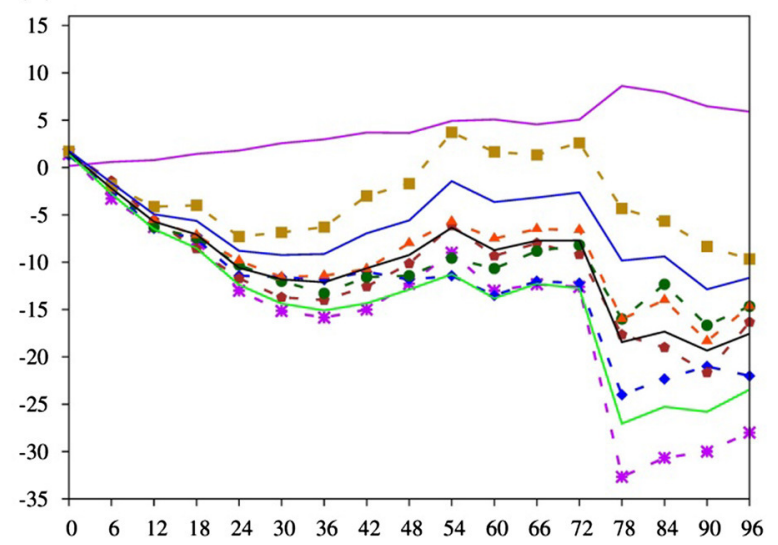

(d)

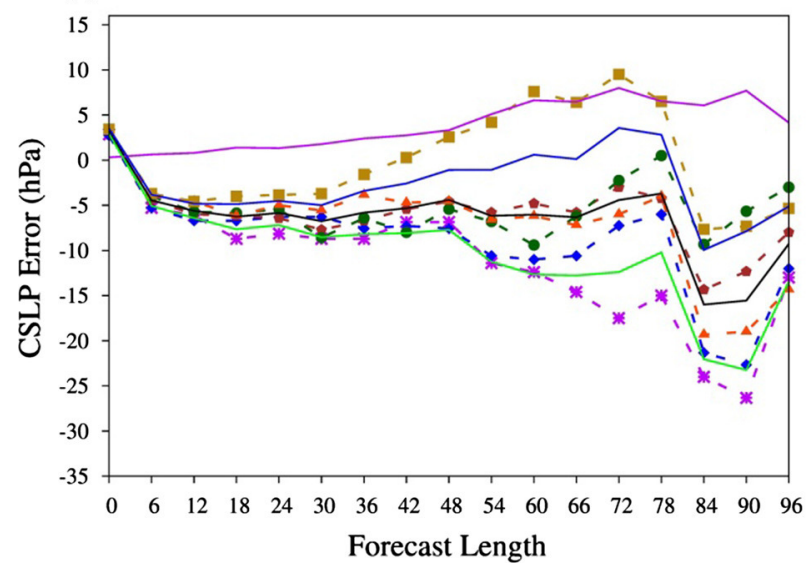

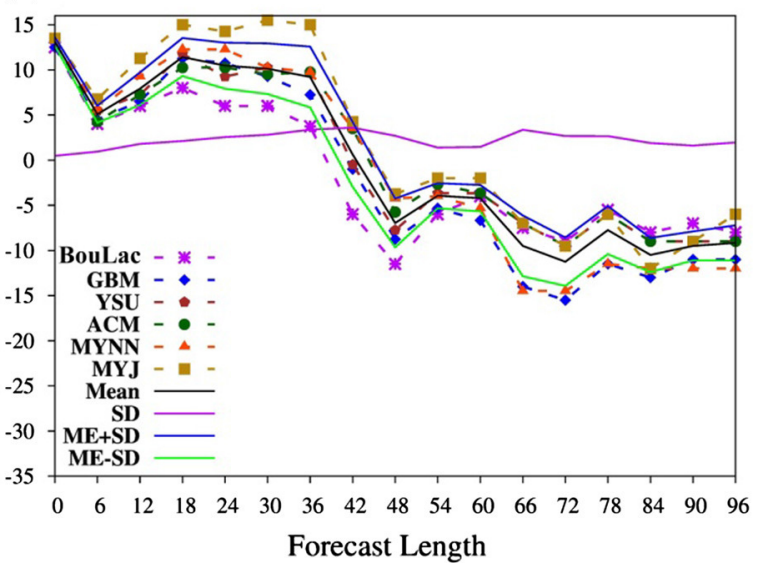

Figure 4

Same as Fig. 2 but for CSLP

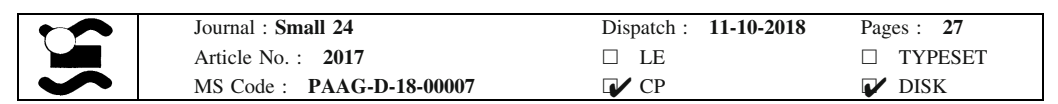


(a)

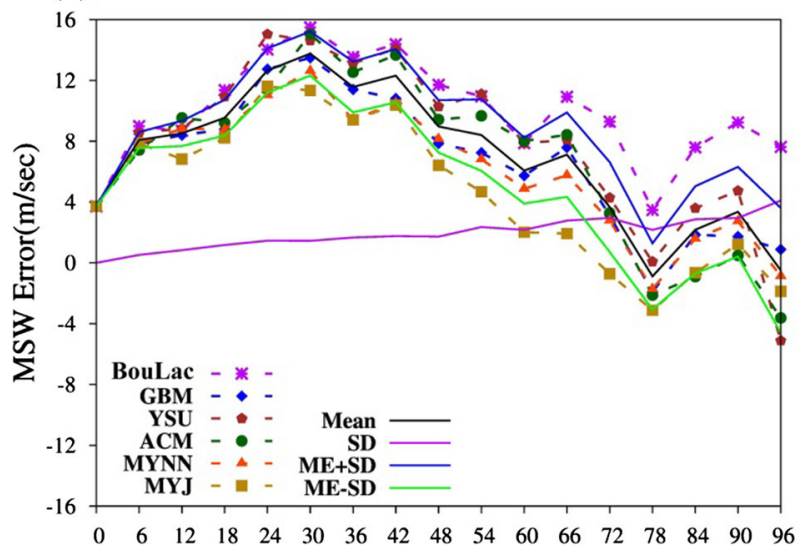

(c)

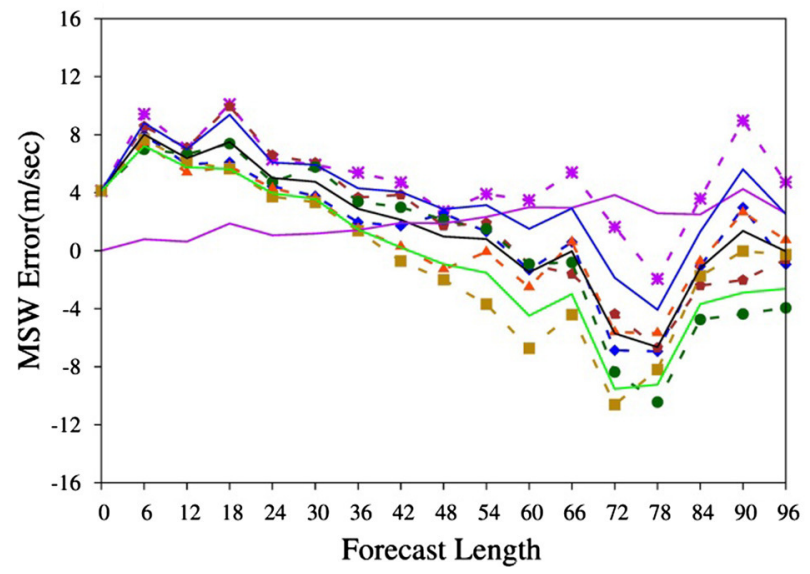

(b)

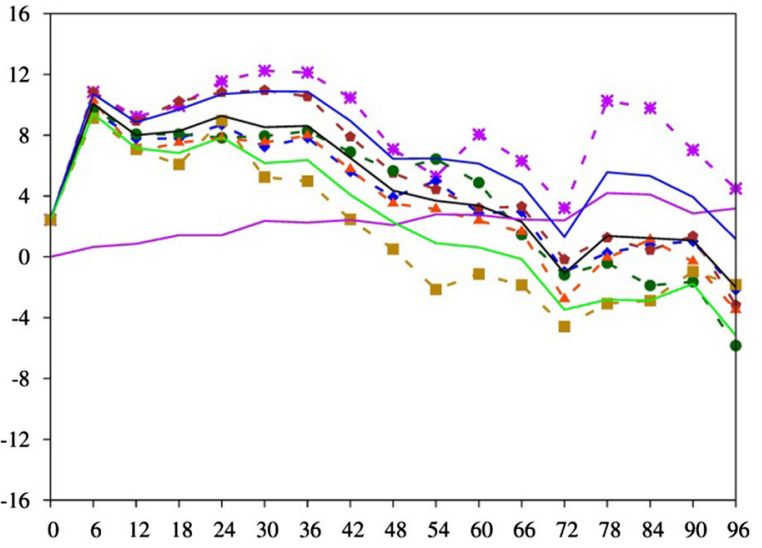

(d)

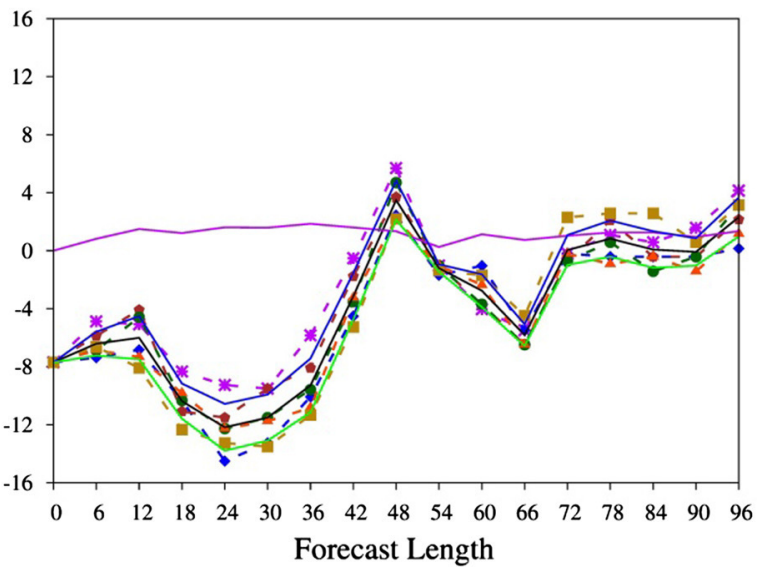

Figure 5

Same as Fig. 2 but for MSW

512 the model forecasts are over-estimated, except the 513 model initialized at the VSCS stage. The PBL 514 scheme-simulated winds are stronger than the 515 observed when the model was initialized from DEP, 516 DD and CS stage till it reached the highest intensi517 fication stage.

518 The time variation of CSLP from the VSCS stage 519 model initialization suggests that the mesoscale 520 model produces less intensity than observed and the 521 model has a tendency to overestimate the intensity 522 after $36 \mathrm{~h}$ of simulation. A similar pattern was also 523 observed in the ME of MSW (Fig. 5) calculated 524 against the IMD observations. The ME and SD of 525 CSLP and MSW suggest that MYJ followed by 526 BouLac schemes produced relatively lower errors in 527 found to be high with MYJ scheme, the intensity pattern of the storm was well simulated, closely agreeing with the conclusion from the earlier work by Kanase and Salvekar (2015). They indicated that track and the translation movement of the TC was captured well by the YSU scheme, while intensity was reproduced well with the MYJ scheme as this scheme doesn't allow mixing of converged surface air above the PBL, resulting in slow transport of moisture within the entrainment layer. The BouLac scheme produces higher storm intensity than the observation in all stages. However, the non-local and first-order closure schemes YSU and ACM depict slightly higher magnitude of the MSW than the MYJ and GBM schemes. These changes in the intensities with different PBL schemes are attributed to the

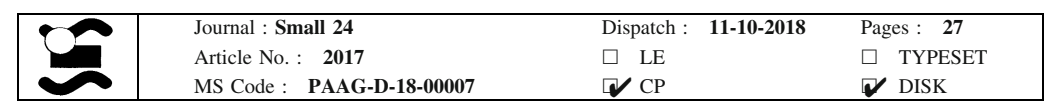


544 differences in the estimation of turbulence and 545

surface drag coefficients employed in the local and non-local turbulence approaches (Hill and Lackmann 2009; Srinivas et al. 2013). The drag coefficients in local schemes is estimated from predictive TKE and length scale, while non-local PBL schemes derive it by making use of the friction velocity and PBL height. From the analysis of seven cyclones, it has been found that the Phailin TC is the best-simulated storm with the least errors in all parameters followed by Hudhudh, while other cases have moderate to large errors.

\subsection{PBL Sensitivity Analysis for Phailin}

In the previous section, we have primarily discussed the quantitative response of boundary layer schemes on the model initialization time during a TC. To analyse exclusively the behaviour of different PBL schemes in simulation of the dynamical and intensification process of TC, we considered TC Phailin as the best case. TC Phailin was an intense storm with an estimated CSLP of $940 \mathrm{hPa}$ and MSW of 115 knots which formed over the BOB after the Orrisa super-cyclone (formed in 1999). The quantitative performance analysis reveals that the response of the PBL schemes with the different stages of model initializations reveal TC Phailin is the best case with minimum errors. In this section, the strength of the cyclone with different PBL schemes is analysed by making use of intensity parameters such as CSLP, MSW, RMW, radial structure of the TC, surface wind flow and accumulated rainfall during landfall of the storm.

\subsubsection{Changes in Simulated Track and Intensity Parameters}

The time variation of vector track error (VTE) produced with six PBL schemes with the model initialized at all four TC stages is shown in Fig. 6. The initial storm position at DEP and DD stages is away from the observed position by $30-40 \mathrm{~km}$ and so the model-simulated tracks by different PBL schemes are deviated to the northeast of the IMD estimates and track errors gradually increased throughout the life cycle. The VTE spread ranging from 20, 50, 100 and $250 \mathrm{~km}$ in the DEP stage and 20,60, 50 and $50 \mathrm{~km}$ in the DD stage at 24, 48, 72 and $96 \mathrm{~h}$, respectively, indicate the VTE simulated by the model is found to be higher in the DEP stage Also, there is clear difference between the simulated track errors by local (MYJ, BouLac, GBM and MYNN) and non-local (YSU and ACM) turbulence PBL schemes. In case of DD stage (Fig. 6b), the magnitude of the track errors is reduced compared to the DEP stage, and the spread of track errors between the schemes is found to be constant after the simulated storm reached its mature stage. In contrast, the model track initialized at the CS stage is identical (Fig. 6c) to the observed storm and track errors are also less than $100 \mathrm{~km}$ with all the PBL schemes. Even though the VSCS shows the minimal track error and spread before landfall, the increase in track error and more spread between the PBL schemes is noticed after landfall (after $54 \mathrm{~h}$ ). The significant reduction of errors in both CS and VSCS stages could be due to the presence of a well-established cyclonic vortex, and the position of model storm centre is very near to the observed storm in the model analysis. Thus, when the model is initialized at the DEP stage, VTE is found to be minimum for MYJ and maximum with ACM and YSU at almost all forecast times, while converse results are observed when the model is initialized at DD and higher stages of the storm. The other three PBL schemes (MYNN, GBM and BouLac) produced intermediate errors between MYJ and ACM. As seen in the quantitative track error analysis (Sect. 4.1), the track error is found to be higher for the non-local schemes compared to the local turbulence schemes, suggesting that the translation movement of the simulated storm is found to be faster in non-local schemes compared to local turbulence schemes when the model initialization is at the DEP stage. When moving the model initialization close to the advanced TC stage, the errors in the translation movement of storm are significantly reduced and also non-local schemes perform better in producing minimum VTE compared to local schemes. Thus, when we initialize the model with a meagre cyclonic vortex, local turbulence schemes perform better than non-local and vice versa when we initialize the model with a well-defined cyclonic vortex.

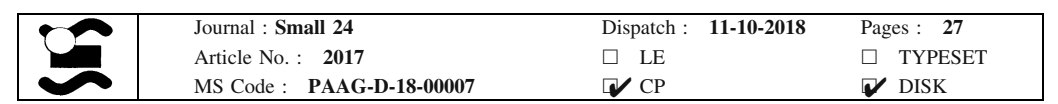




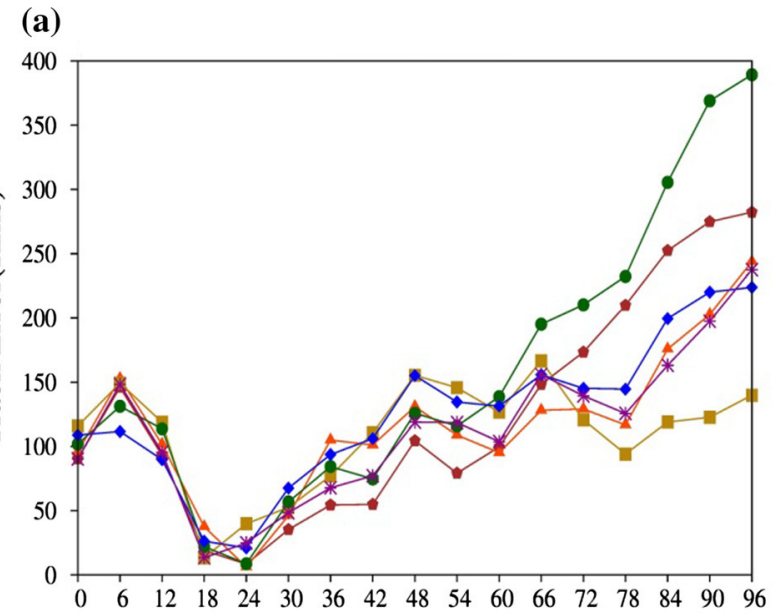

(c)

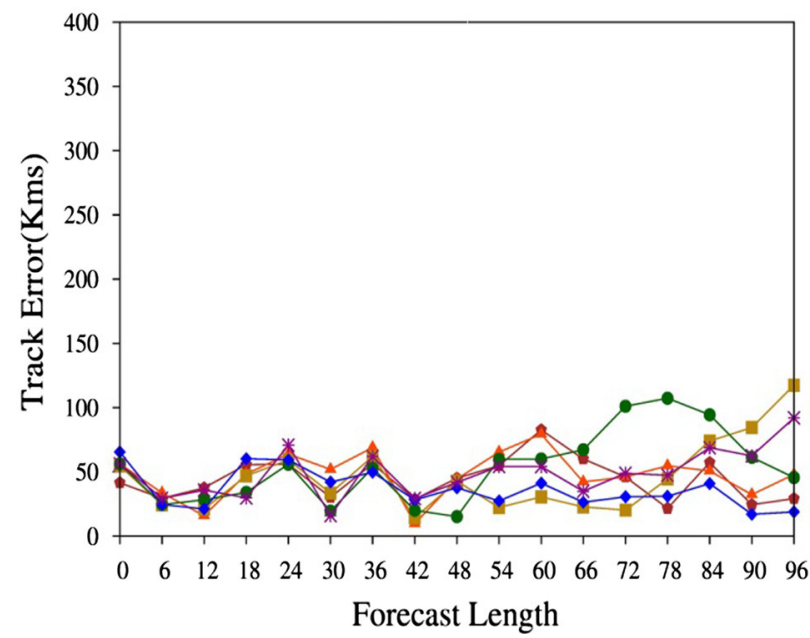

(b)

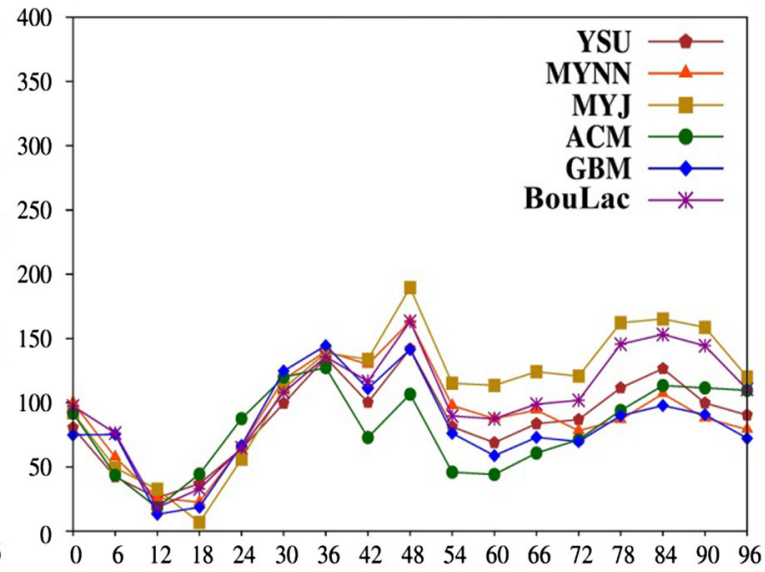

(d)

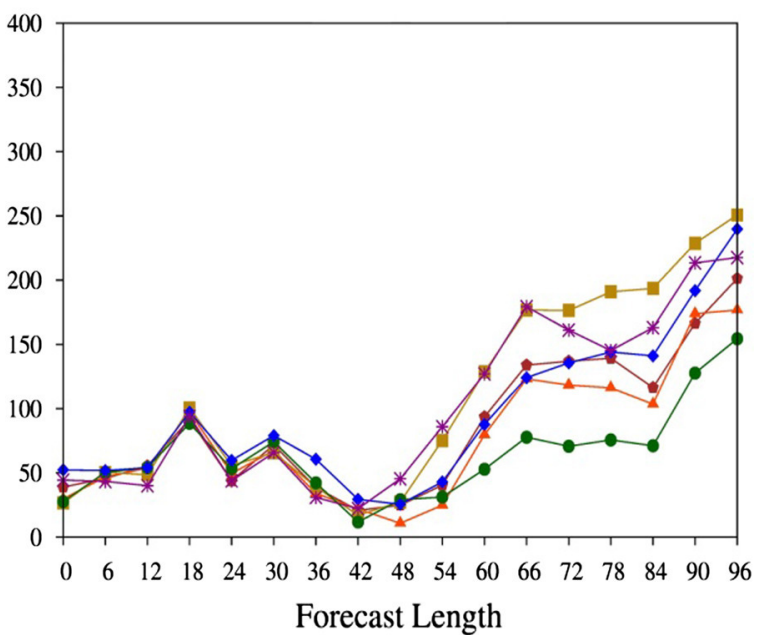

Figure 6

Time variation of vector track error for TC Phailin with the model initializations at four TC stages: a DEP, b DD, c CS and d VSCS

The time series comparison of simulated CSLP and MSW along with IMD estimates for TC Phailin is shown in Figs. 7 and 8, respectively. At the DEP stage (Fig. 7a), the model simulates lower CSLP and higher MSW compared to IMD in the first $36 \mathrm{~h}$ of simulation, and intensity differences are found to be less sensitive with the PBL schemes. However, the intensity starts to increase during the rapid intensification phase of the modelled storm and larger difference is found when the model reaches its mature stage. Interestingly, the rapid intensification is clearly reproduced by all the schemes from 42 to $72 \mathrm{~h}$ of simulation even though the model is initialized before developing stages of the storm. A similar kind of response is observed when the model is initialized at the DD (Fig. 7b) stage, but the intensification rate is found to be high till $36 \mathrm{~h}$ of simulation and also it is seen that the weakening phase of the storm after $84 \mathrm{~h}$ of model integration is well simulated by all PBL schemes. When the model is initialized with CS and VSCS stages (Fig. 7c, d), all the PBL schemes nearly captured all three phases (deepening, mature and weakening) of the storm close to observed intensities. Moreover, the rapid intensification of storm both in term of its rate and duration was simulated well with all PBL schemes; however, the major difference was found during the mature phase of the storm. The BouLac scheme produced higher intensity while the

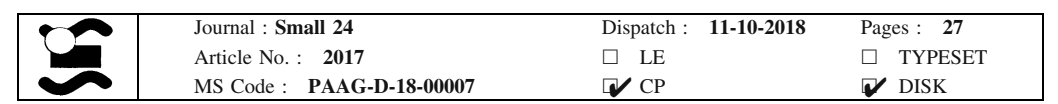


(a)

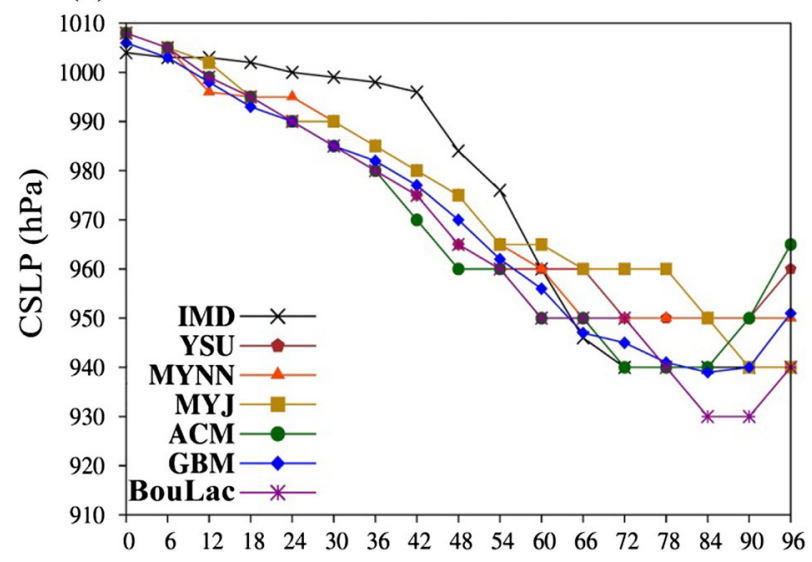

(c)

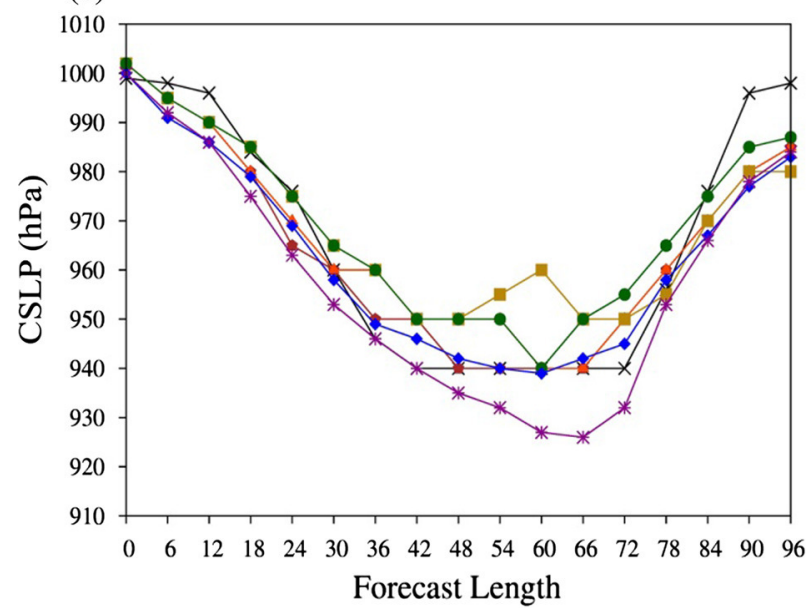

(b)

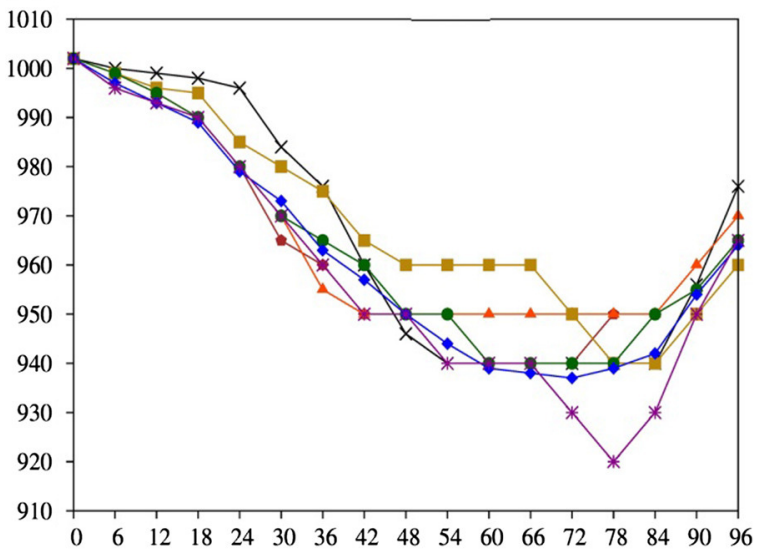

(d)

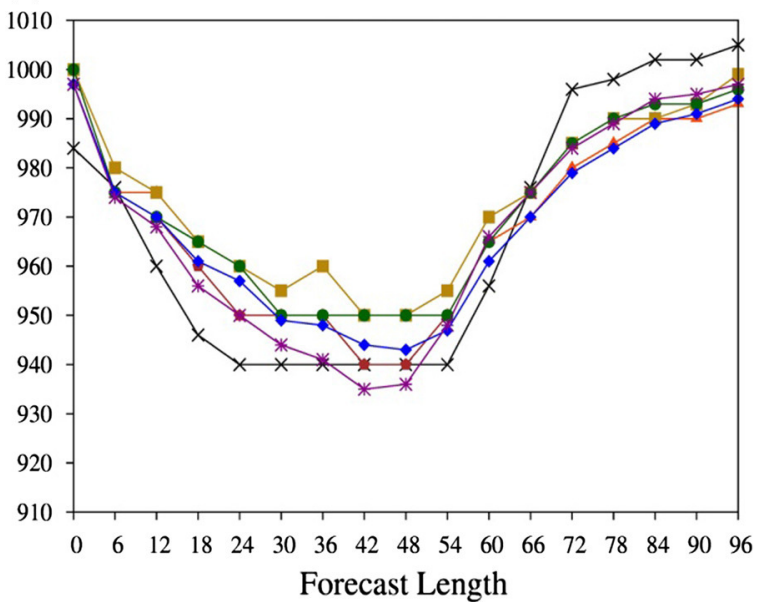

Figure 7

Same as Fig. 6 but for CSLP

MYJ scheme simulated weaker intensity; only the YSU and GBM schemes captured intensity closer to the observed. The deepening phase, as seen in simulated CSLP, when the model initialized at VSCS, is found to be longer (30-42 h) than the observed duration and it could be due to the large pressure difference between the model and observed storm at the time of model initialization. As noticed in the tracking error, the MYJ PBL scheme produced CSLP with higher errors and ACM and BouLac exhibited minimal errors in intensity than the other schemes. Similar behaviour of intensity structure is also observed as in the case of time evaluation of MSW (shown in Fig. 8). However, the intensity of MSW was over-estimated by all the schemes in the initial phase of simulation (36 h); furthermore, MSW was 677 underestimated by $5-15 \mathrm{~m} / \mathrm{s}$ during simulation of the mature phase at all stages of model initialization. The maximum underestimation of winds was found with all the PBL schemes when the model was initialized at the VSCS stage. The model with the BouLac PBL scheme produced the stronger winds at the observed intensification rate, except with an initial DEP condition; meanwhile, MYJ scheme produced relatively weaker winds compared to the other four PBL schemes. RMW, which indicates the intensity of a cyclone and the size of the cyclone vortex, is the distance between the centre of the storm and the maximum winds of the eye wall; it is one of the crucial parameters that needs to be understood. The

\begin{tabular}{|l|lll|}
\hline & Journal : Small 24 & Dispatch : 11-10-2018 & Pages : 27 \\
& Article No. : 2017 & $\square$ LE & $\square$ TYPESET \\
& MS Code : PAAG-D-18-00007 & $\square \mathrm{CP}$ & $\checkmark$ DISK \\
\hline
\end{tabular}



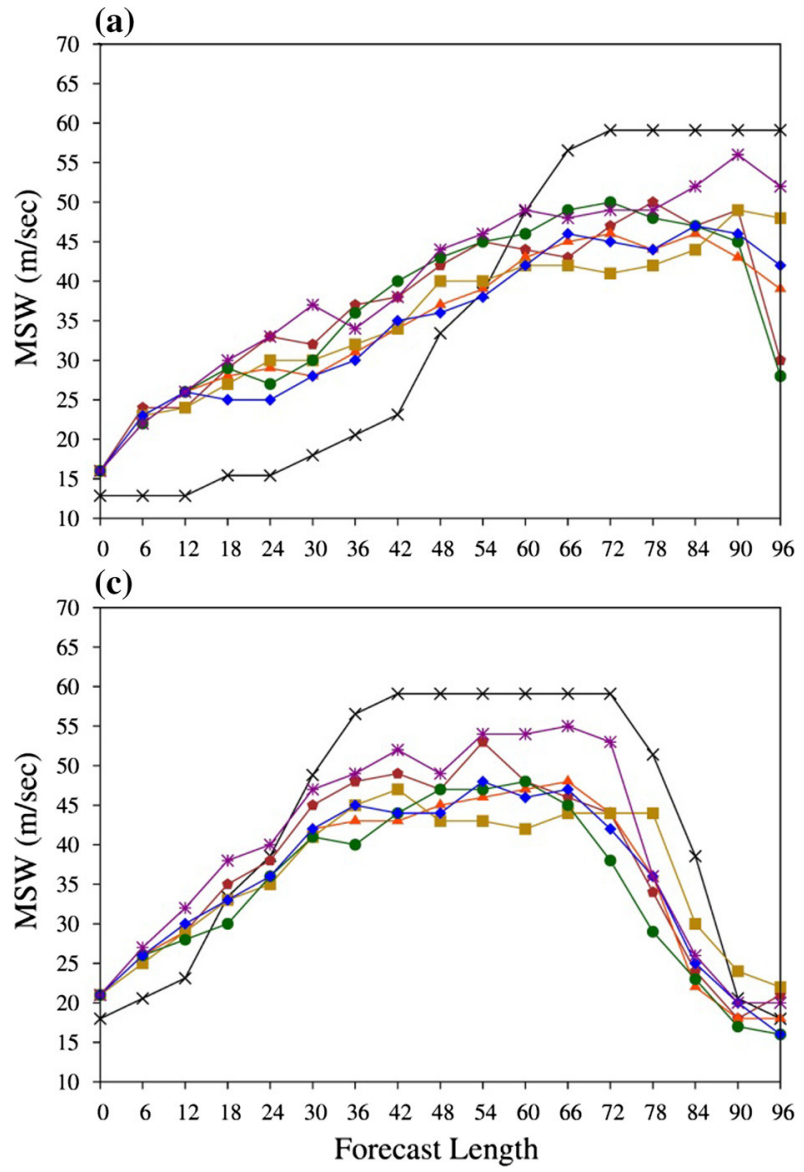

(b)

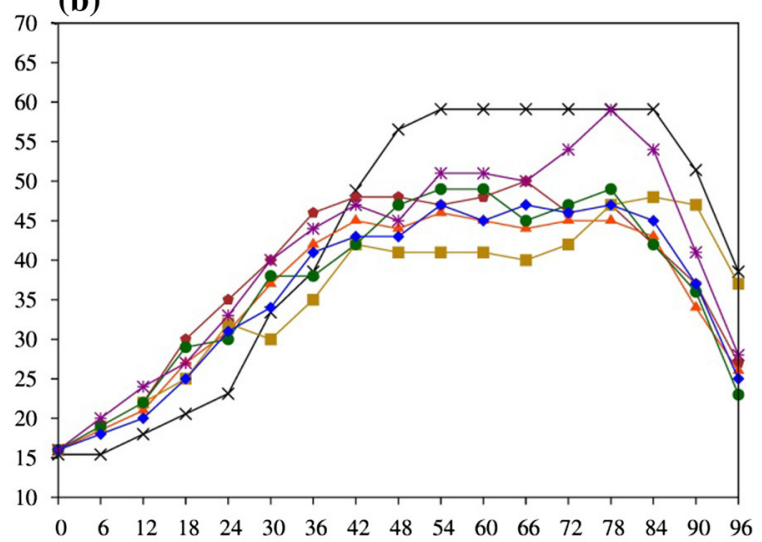

(d)

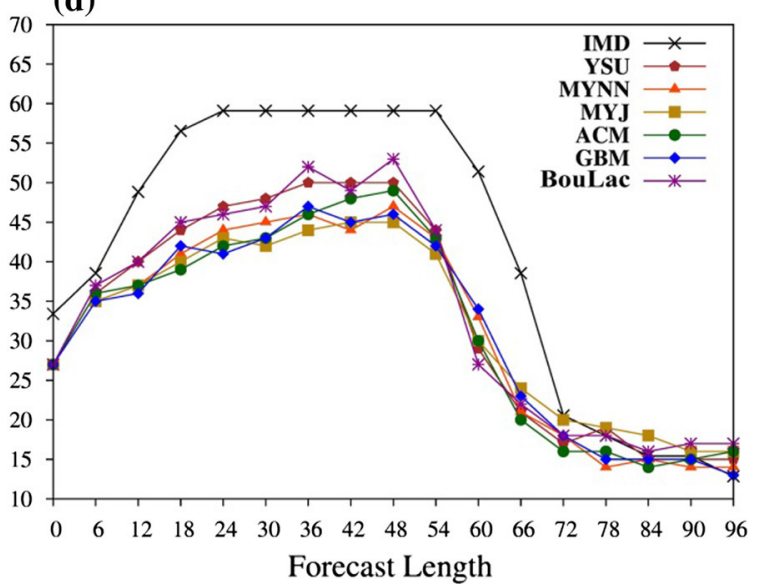

Figure 8

Same as Fig. 6 but for MSW

RMW calculated for the model simulations with four stages of initialization are compared with corresponding observations of CIRA (Fig. 9). In the DEP stage, the model with all the PBL schemes simulated RMW close to observed CIRA estimates during the rapid intensification phase, but when the model storm reaches its mature phase after $78 \mathrm{~h}$ of simulation, the simulated RMW difference was found to be high. In contrast, with the model initialized at the DD stage, the simulated RMW is nearly same as observed and a sudden raise is noticed during the weakening phase of the storm between 90 and $96 \mathrm{~h}$ of model simulation; this could be due to inability to simulate the observed intensities after crossing the land surface. However, with CS and VSCS stages, the RMW obtained from PBL schemes is over-estimated, particularly during the weakening phase of the storm, indicating a larger cyclone size simulated by the model than CIRA. The RMW simulated by the BouLac scheme is close to the observations when the model is initialized at all stages of TC, except the weakening phase of the storm with CS and VSCS initializations (Fig. 9c, d). At all stages of model initialization, the RMW spread between the PBL schemes is increasing with the forecast lead times, particularly when the model is simulated as a CS and VSCS, indicating the large sensitivity during weakening of the storm after landfall; this could be due to differences in simulation of drags and mixing coefficients in turbulence schemes. Therefore, the results of RMW parameter are in line with the intensities of CSLP and MSW.

Thus, if we consider the model PBL sensitivity at the DEP stage of the storm, local PBL schemes predicted the simulated storm with intensity higher

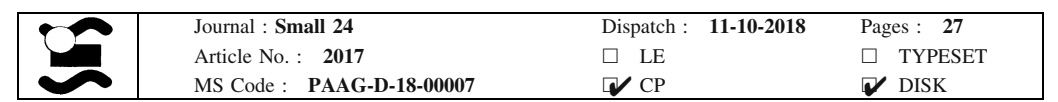




\section{(a)}

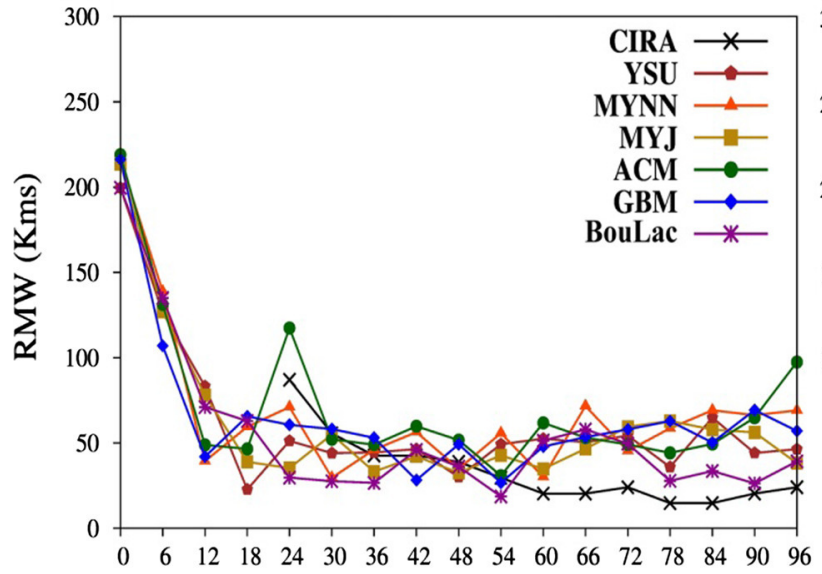

(c)

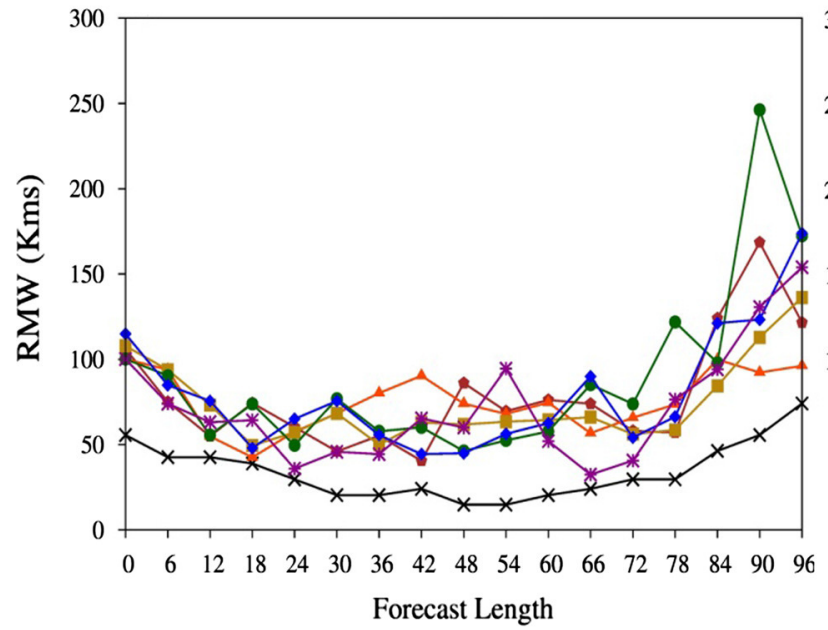

(b)

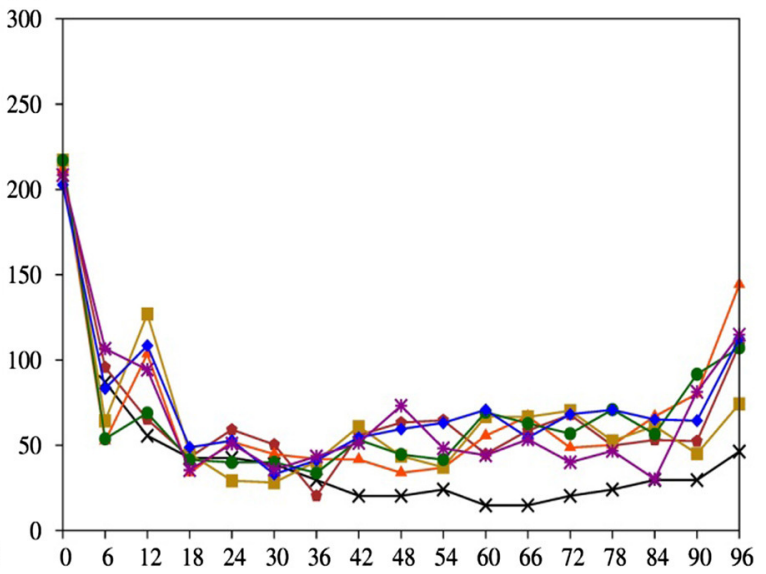

(d)

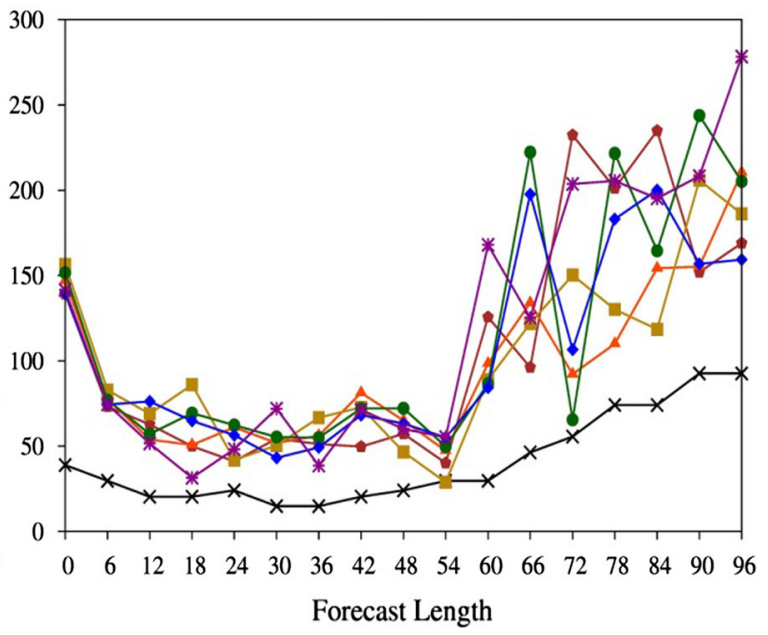

Figure 9

Same as Fig. 6 but for RMW

than the observed. However, the local schemes produced a less intense storm when we shifted the model initialization to a mature TC stage. One of major causes for the less intensity in local schemes could be due to the tendency of the local schemes to simulate shallow PBL heights compared to non-local schemes (Xie et al. 2012); an additional cause may be the formation of the statically stable layers during the growth of PBL, which inhibits the growth of PBL into deeper heights (Cohen et al. 2015). Among local schemes, the simulation of highest intensification with the BouLac scheme is due to high and welldefined eddy diffusion coefficients which can simulate higher turbulence levels and transfer momentum into deeper layers (Bougeault and Lacarrere 1989). The intensity changes observed with local and nonlocal PBL schemes can influence the simulation of vertical TC structure. To illustrate the clear impact, we have analysed the radial-height cross-section of wind and temperature anomaly by considering the best-performing case (BouLac) against one non-local (YSU) and local PBL scheme (MYJ).

\subsubsection{Radius-height Wind Structure of TC Phailin}

The radius-height cross section of the azimuthally averaged gradient wind and its simulated differences between the local and non-local scheme against the

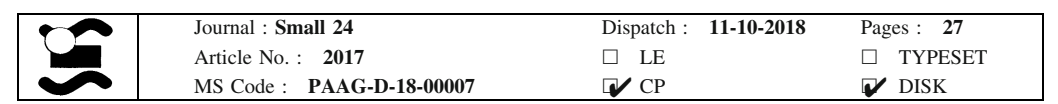



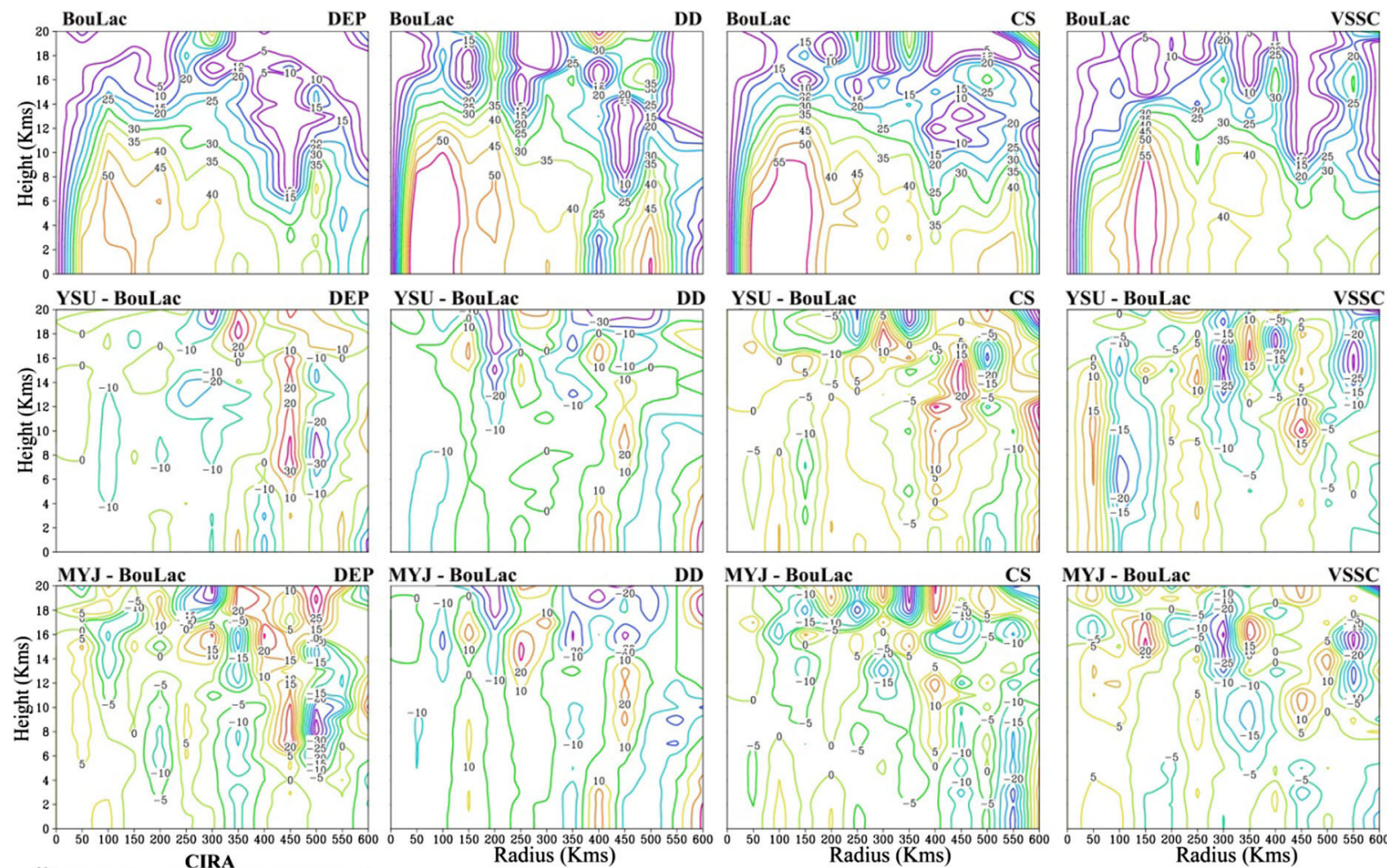

DD MYJ - BouLac
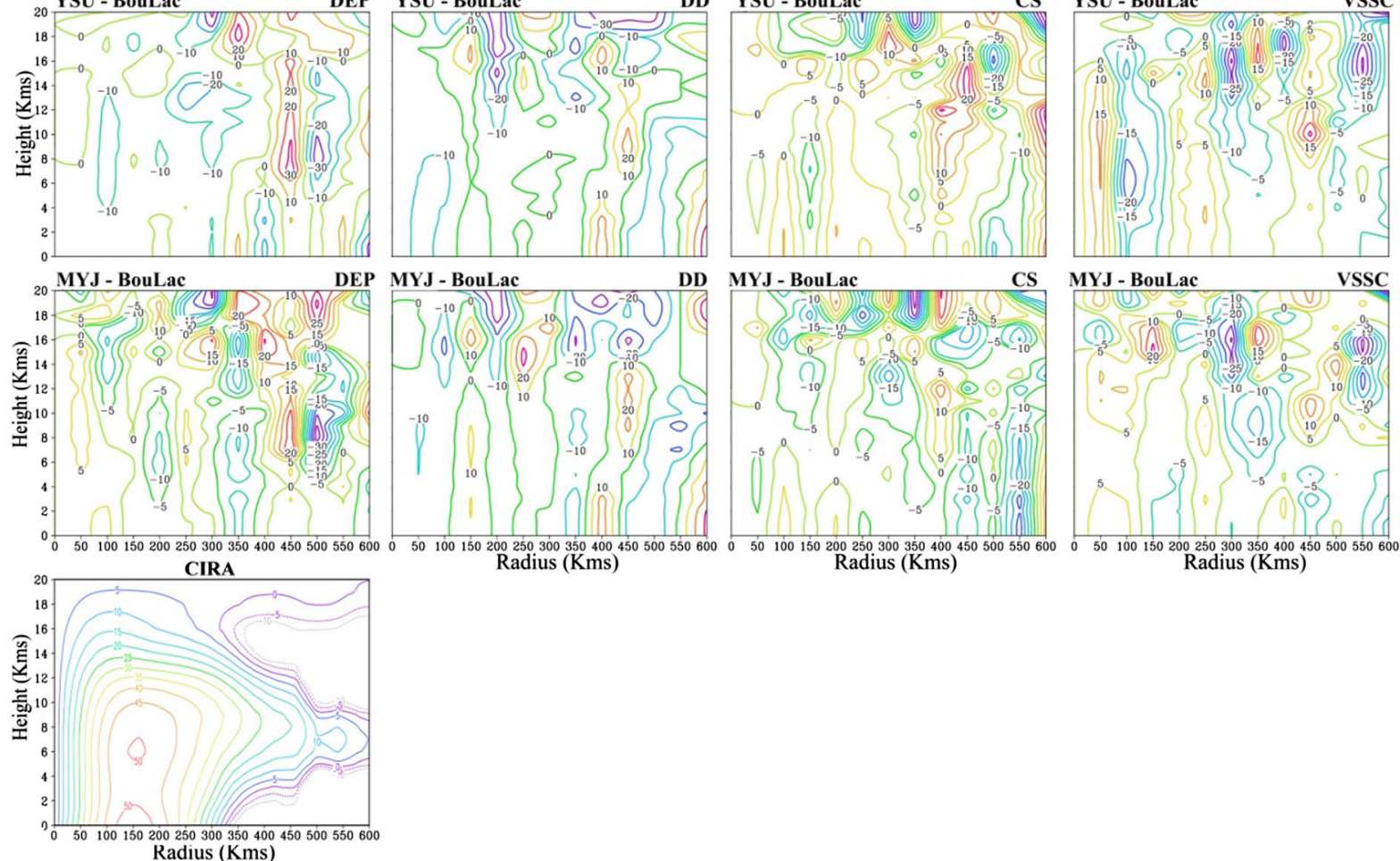

Figure 10

Comparison of azimuthally averaged radius-height cross section of gradient wind (knots) obtained from CIRA with the BouLac scheme and its differences with the local (MYJ) and non-local schemes (YSU) on 1200 UTC, 12 October 2013

best-performing scheme (BouLac) are plotted at a mature TC stage, i.e., on 1200 UTC of 12 October 2013, as shown in Fig. 10. The gradient wind estimates obtained from CIRA indicate the band of calm winds in the eye of the storm is narrow (20-25$\mathrm{km}$ ) and the strongest winds (40-50 knots) extended vertically from the surface to $10 \mathrm{~km}$ within the radius of 90-220 km from centre of the storm; furthermore, the wind strength in the eye wall region is reducing gradually with vertical and horizontal distances of 10 and $200 \mathrm{~km}$, respectively, from the eye of the storm. Though the magnitude of maximum gradient wind is more or less similar in all three experiments, the horizontal extensions of the strong wind region (50 knots) differ in the three PBL schemes. The BouLac scheme initialized at various stages shows the presence of the strongest winds up to $10 \mathrm{~km}$ in a radius of $50-220 \mathrm{~km}$ from the eye of the storm, except during the DEP stage where the maximum winds do not exceed beyond $8 \mathrm{~km}$ from the surface, indicating moderate intensities simulated in the DEP stage. Although the vertical distribution of the maximum wind is not simulated in the MYJ PBL scheme at the DEP stage, the horizontal extension of maximum winds (50 knots) is well simulated with less deviation from CIRA. Also, when we move

\begin{tabular}{|l|lll|}
\hline & Journal : Small 24 & Dispatch : 11-10-2018 & Pages : 27 \\
& Article No. : 2017 & $\square$ LE & $\square$ TYPESET \\
MS Code : PAAG-D-18-00007 & $\square \mathrm{CP}$ & $\checkmark$ DISK \\
\hline
\end{tabular}


778 model initialization towards deepening stages of the 779 storm, there is a gradual increase in the gradient 780 winds in terms of spatial extent and intensity, 781 indicating the enhancement of inner core/eye wall 782 region of the storm. Also, the strongest gradient 783 winds simulated with the model initialized at an 784 advanced stages of the storm are in the order of 55 785 knots indicating all the PBL schemes slightly over786 787 788 789 790 791 792 793 794 795 796 797 798 slightly extended (nearly 100-km) in simulations of MYJ and the best comparison (70-80-km) is found with the BouLac scheme. Thus, the vertical and horizontal distribution of gradient winds with the BouLac scheme represented more accurately the observed, except in the DEP stage, while the MYJ scheme produced relatively less deviations and observations close CIRA in the DEP stage.

The radius-height sections of the radial wind (contour) and vertical velocity fields (shaded) at the mature phase of the storm (1200 UTC of 12 October 2013) are shown in Fig. 11. The radial wind pattern shows the strong, low-level inflow $(-20 \mathrm{~m} / \mathrm{s})$ nearly extended up to the radius of $100 \mathrm{~km}$ from the outer eye wall and spiral band regions of the storm and the strong upper tropospheric outflow (12-16-km) simulated with all PBL schemes. Strong upward motions (maximum of $1.4 \mathrm{~m} / \mathrm{s}$ ) around $50-100 \mathrm{~km}$ radius from surface to $14-\mathrm{km}$ height are noticed with all the PBL schemes (Fig. 11), indicating that the model predicted ascending motions over the core region and
799

800

801

802

803

804

805

806

807

808

809

810

811

812

813

814

815

816

817

818

819
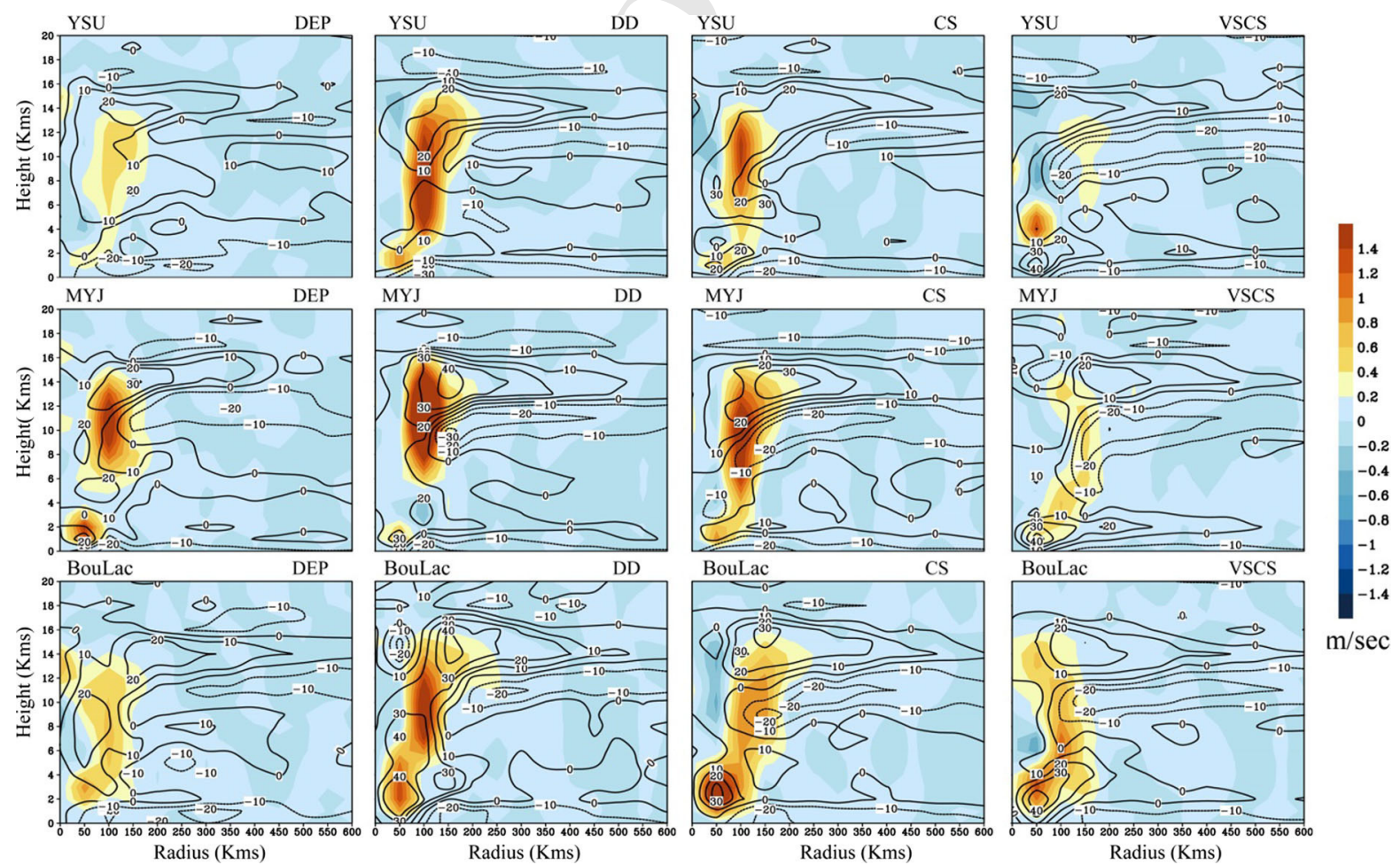

Figure 11

Distribution of azimuthally averaged radius-height cross section of simulated radial wind (contour) and vertical velocity (shaded) around TC Phailin at 1200 UTC 12 October 2013

\begin{tabular}{|l|lll|}
\hline & Journal : Small 24 & Dispatch : 11-10-2018 & Pages : 27 \\
& Article No. : 2017 & $\square$ LE & $\square$ TYPESET \\
& MS Code : PAAG-D-18-00007 & $\square \mathrm{CP}$ & $\checkmark$ DISK \\
\hline
\end{tabular}


820 descending motions ( -0.4 to $-0.8 \mathrm{~m} / \mathrm{s}$ ) in the eye 821 region, indicating the strength of the secondary 822 circulation. However, the YSU scheme has predicted 823 weaker inflow winds and resulting ascending motion 824 compared to other schemes, indicating that the YSU 825 scheme failed to simulate the secondary circulation of 826 the storm. The higher radial and vertical velocities 827 simulated by the MYJ scheme indicates the better 828 829 830 831 832 833 representation of secondary circulation leading a high-intensity storm compared to the other PBL schemes. As seen in the gradient winds, when we move model initialization towards the advanced stages of the storm, there is a gradual increase in both radial and vertical winds, indicating the strength of secondary circulation is increasing with the advanced stages of the storm.

\subsubsection{Vertical Temperature Structure of TC Phailin}

The radius-height section of the azimuthally averaged temperature anomaly at the mature phase of the storm is shown in Fig. 12. The anomaly in temperature is computed as a deviation of temperature fields before progression of the storm to its mature stage (1200 UTC 12 October 2013). The temperature anomaly obtained from CIRA indicates that the maximum warming of $5{ }^{\circ} \mathrm{C}$ occurs between 10 and $13 \mathrm{~km}$ of height with radius of 80 to $100 \mathrm{~km}$, while the
834 835

\section{6}

837

838

839

840

841

842

843

844

845
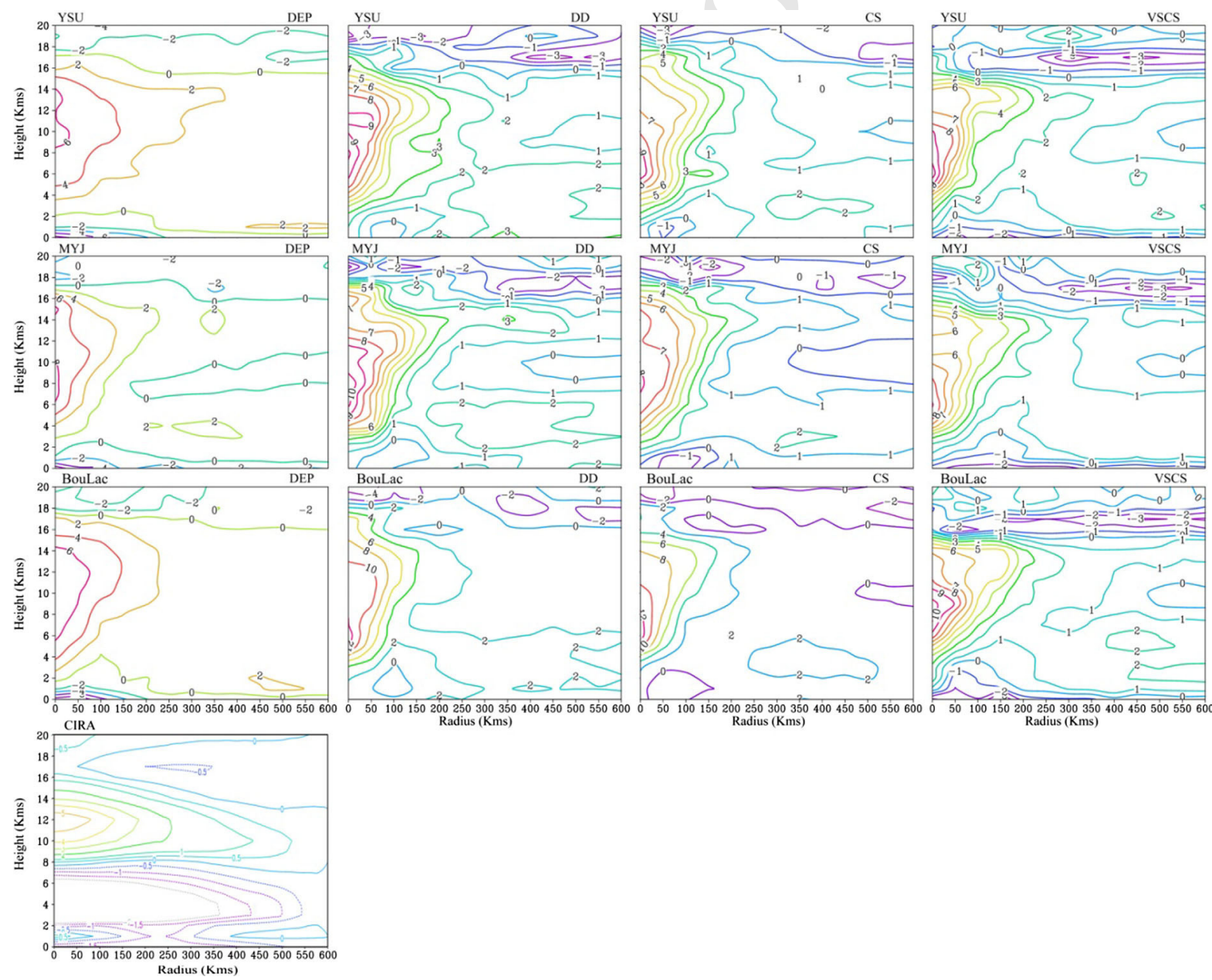

20025030035040
Radius (Kms)

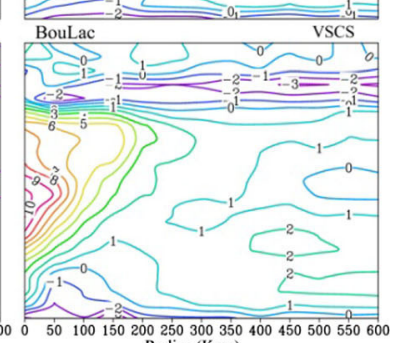

200250300350
Radius (Kms)

Radius (Kms)

Figure 12

Depiction of azimuthally averaged radius-height cross section of simulated temperature anomalies from BouLac, YSU and MYJ PBL schemes with CIRA for TC Phailin at 1200 UTC 12 October 2013

\begin{tabular}{|l|lll|}
\hline & Journal : Small 24 & Dispatch : 11-10-2018 & Pages : 27 \\
& Article No. : 2017 & $\square$ LE & $\square$ TYPESET \\
MS Code : PAAG-D-18-00007 & $\square \mathrm{CP}$ & $\checkmark$ DISK \\
\hline
\end{tabular}


846 maximum cooling was noticed at a range of 3-6 km 847 in height, indicating strong convergence and associ848 ated evaporation results in cooling at lower levels and 849 the presence of strong outflow region resulting in the 850 subsidence of air and warming at the eye of the storm 851 in the mid-upper troposphere levels. Meanwhile, the 852 model-simulated temperature anomaly with all PBL 853 schemes indicates maximum warming of $6-10{ }^{\circ} \mathrm{C}$ at 854 855 856 857 858 a height of $6-12 \mathrm{~km}$ spread over $50-80 \mathrm{~km}$. The warming-related temperature anomaly is found close to the observed value $\left(6^{\circ} \mathrm{C}\right)$ when the model is initialized at the DEP stage, while the anomaly increases up to $10{ }^{\circ} \mathrm{C}$ when the model is initialized at advanced storm stages. Also, the magnitude of the warming differs from scheme to scheme; maximum warming of $6,9,10$ and $10{ }^{\circ} \mathrm{C}$ is found at DEP, DD, $\mathrm{CS}$ and VSCS stages, respectively, in YSU and BouLac schemes. There is an increase in the magnitude of the warming in non-local schemes when model initialization move towards an advanced TC stage, while the MYJ model exhibits a slightly different trend in the magnitude of warming. The MYJ scheme exhibits increased warming from DEP to DD $\left(8-10{ }^{\circ} \mathrm{C}\right)$ and a further decrease in temperature anomaly $\left(8-6^{\circ} \mathrm{C}\right)$ at later stages, suggesting that the strength of circulation and warming were decreasing in the local MYJ scheme when the model was initialized in advance storm stages. Considering the error limits for CIRA (SD nearly $3{ }^{\circ} \mathrm{C}$ ), the overestimation of simulated core warming clearly noticed in the advanced stages of the model initialization is negligible for the MYJ scheme (less than $0.5{ }^{\circ} \mathrm{C}$ ) and seems to be significant in case of the BouLac and YSU schemes which are in the order of 1 and $2{ }^{\circ} \mathrm{C}$, respectively. Therefore, as mentioned regarding the gradient wind, the PBL schemes simulated the storms with high intensity when the model was initialized at advanced stages of the storm. This could be due to the variations in the estimation of turbulence drag coefficients between local and non-local diffusion schemes. The difference in turbulent coefficients will primarily affect the surface wind flow (Srinivas et al. 2013; Hari Prasad et al. 2015) and associated convergence in the TC inflow region which results in changes in thermo-dynamical processes, producing the different thermal structure of the TC.

\subsubsection{Vertical Distribution of Zonal and Meridional Winds at a Mature Stage}

The model initialization at different TC stages with different PBL schemes produced sizable differences on the simulation of wind profiles at Visakhapatnam (Fig. 13). The differences are found majorly in meridional winds compared to the radiosonde observations at the DEP stage and thereafter the models are well predicted. As the model is initialized at
875

876

877

878

879

880

881

882

883

884

885

886

887

888

889

890

891

892

893

894

895

896

897

898

899

900

901
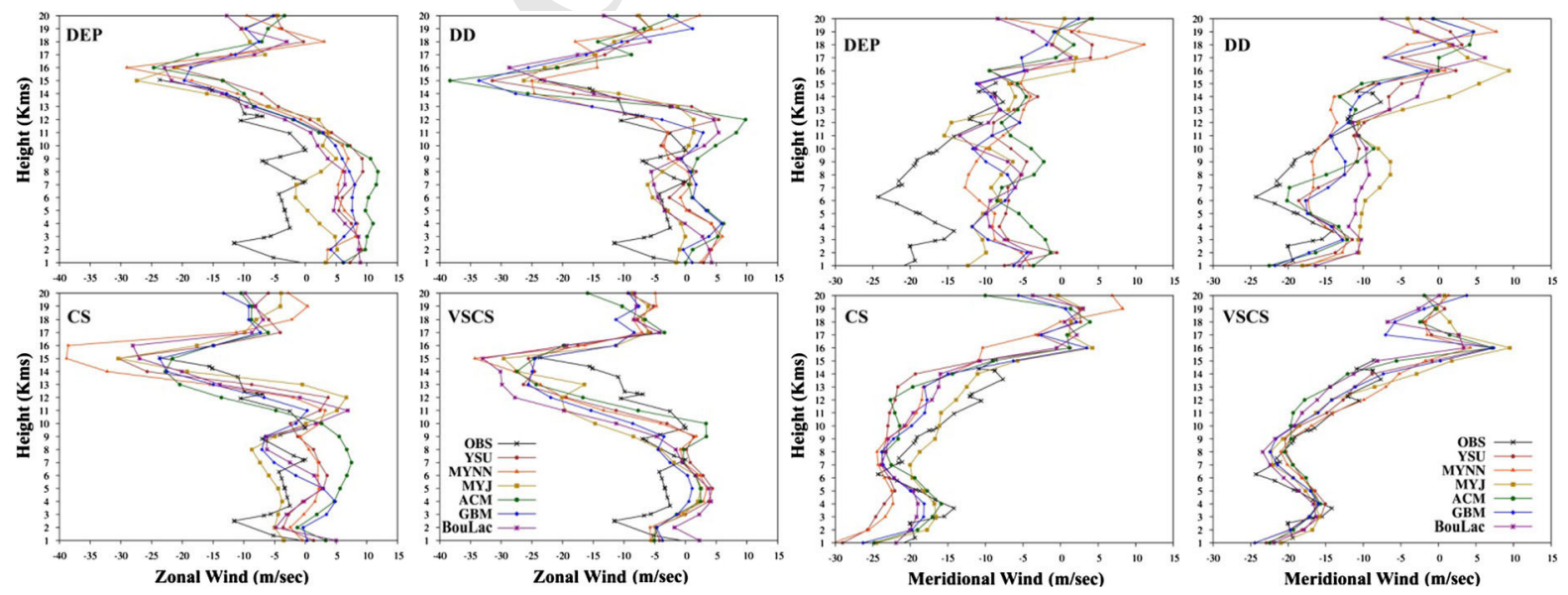

Figure 13

Comparison of zonal (left panel) and meridional (right panel) winds during the mature stage of the cyclone for a radiosonde station, Vishakapatnam at 0000 UTC 12 October 2013

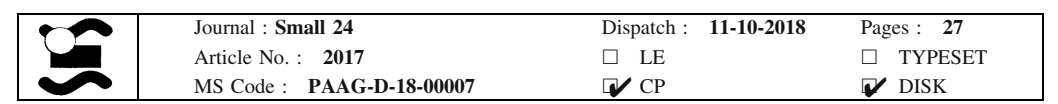


902 advanced stages of the storm, the differences between 903

simulated and observed zonal and meridional winds are matched well. And the winds at upper and surface levels are also simulated close to radiosonde observations by all the schemes at CS and VSCS stages. At DEP and DD stages, the winds simulated by all PBL schemes show the wind flow is associated with a relatively strong westerly component and moderately weak northerly flow, whereas the observations indicate the wind flow is mainly a northerly flow. The possible reasons for model failure in producing winds from surface to the mid-troposphere (up to $11 \mathrm{~km}$ ) is due to the fast translation movement of the simulated storm. A sudden rise in the magnitude of the easterly at $15-\mathrm{km}$ height in the outflow region is well simulated by all the PBL schemes; it is also seen that the model exhibited consistent performance in capturing the upper tropospheric wind pattern in terms of the direction and intensity of the TC outflow region irrespective of model initialization at all the four stages. However, there are considerable fluctuations in simulated zonal wind during the model initialization at CS and VSCS stages. The radiusheight cross-section analysis shows the MYJ scheme exhibits less errors compared to all other five PBL schemes when the model is initialized at the DEP stage, while the non-local PBL schemes perform (YSU and ACM) better during the model initialization at CS and VSCS stages. Out of six PBL schemes, the MYJ scheme produced weaker winds (on average 2-3 $\mathrm{m} / \mathrm{s}$ ) and the BouLac scheme produced stronger winds $(>5 \mathrm{~m} / \mathrm{s})$ when the model was initialized at advanced storm stages which are significantly higher than the errors limits of radiosonde $(0.5 \mathrm{~m} / \mathrm{s})$. These differences in the lower- and upper-level winds arise mainly due to the variations of diffusion coefficients and frictional velocities between the PBL schemes, which could have impacted the lower-level radial wind inflow and upper tropospheric outflow regions of simulated TCs.

\subsubsection{Surface Wind Analysis During Landfall}

To examine the sensitivity of the PBL schemes in simulating the wind pattern during landfall, surface wind flow with different PBL schemes by varying model initialization at four stages of the storm are presented in Fig. 14 along with the multiplatform winds from CIRA. The CIRA winds indicate a strong cyclonic storm with well-established circular symmetry of maximum winds (wind speed between 64 and 119 knots) around the region $17^{\circ} \mathrm{N}-20^{\circ} \mathrm{N}$ and $84^{\circ} \mathrm{E}-87^{\circ} \mathrm{E}$. A similar pattern is also seen with different PBL schemes, except with the BouLac scheme, with less intense cyclonic circulation. The maximum closed isotach in CIRA data clearly indicates that the system has not yet made landfall, but the model initialized during the DEP stage indicates the cyclone crossed the coast. The observations show the maximum winds concentrated at the right rear sector of the observed storm with wind speeds ranging from 80 to 95 knots near the core of the cyclone, while the model-simulated wind is 65-95 knots. To conclude, the MYJ scheme performed well with the DEP stage initialization and the BouLac scheme performed well with initializations at the DD and CS stages.

\subsubsection{Analysis of TC Phailin-Induced Rainfall}

Accurate prediction of the rainfall both spatially and temporally before, during and after landfall is crucial for decision makers for planning activities. The precipitation in tropical storms generally occurs around the wall cloud region of the cyclone due to organization of horizontal winds and clouds in spiral bands around a relatively calm central eye region characterized with subsiding vertical motion. As the maximum surface winds with all the PBL schemes are found to be more or less in right forward and rear sectors of the simulated storm, the maximum rainfall pattern is expected to shift towards the left forward sector of the storm, as mentioned by previous studies (Osuri et al. 2015; Greeshma et al. 2015; Dasari et al. 2017). The gridded rainfall observation of IMD and the satellite-derived rainfall estimates of CMORPH, TRMM and CPC (bottom panel of Fig. 15) indicate the circular distribution of rainfall with heavy rainfall is confined to the left forward or south-west sector of the storm. The maximum rainfall (more than $200 \mathrm{~mm}$ /day) is concentrated over coastal region of Orissa and northern Andhra Pradesh. The rainfall bands of the storm are extended up to north-central Andhra Pradesh.
967

\begin{tabular}{|l|lll|}
\hline & Journal : Small 24 & Dispatch : 11-10-2018 & Pages : 27 \\
& Article No. : 2017 & $\square$ LE & $\square$ TYPESET \\
& MS Code : PAAG-D-18-00007 & $\downarrow \mathrm{CP}$ & $\checkmark$ DISK \\
\hline
\end{tabular}



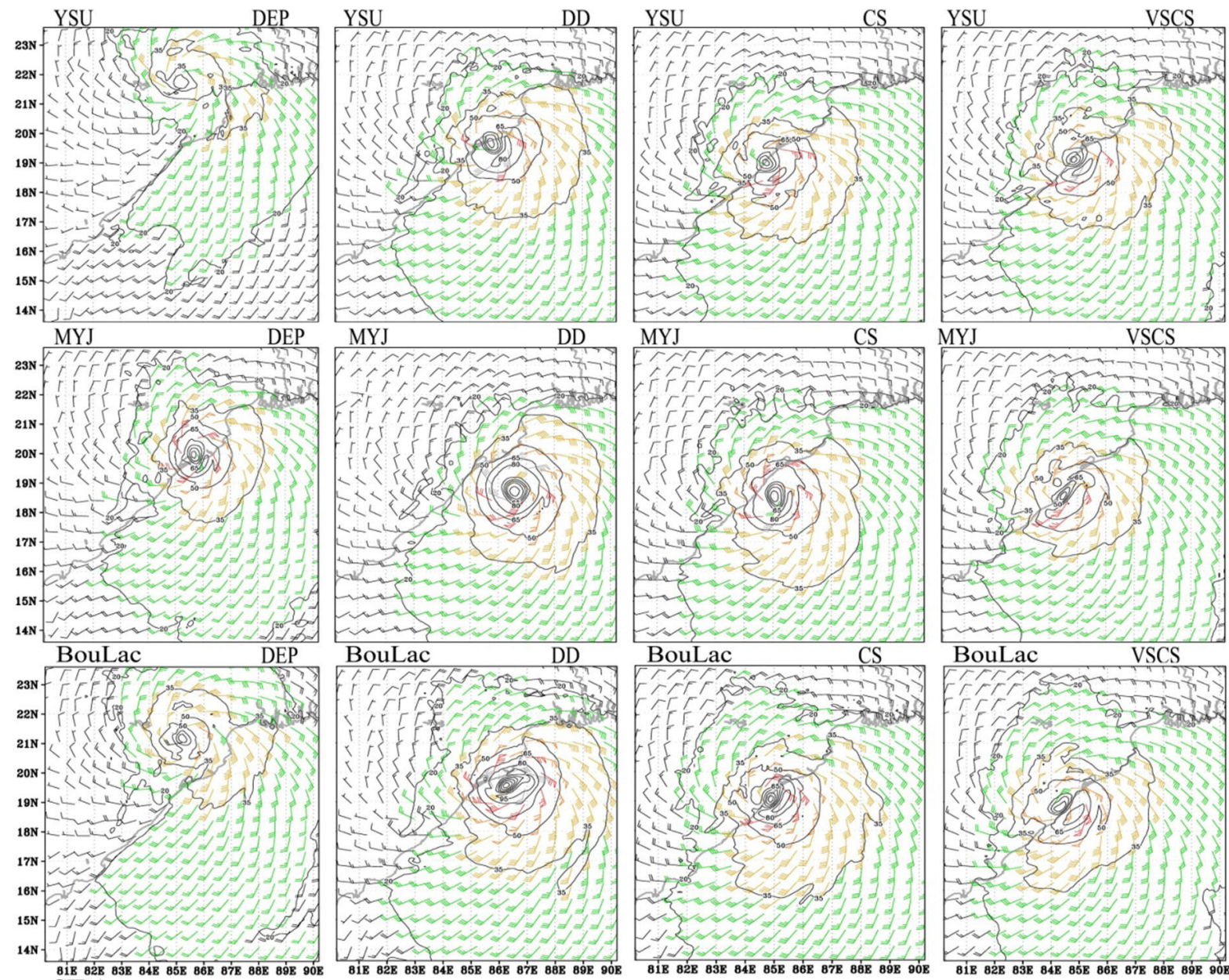

BouLac DD
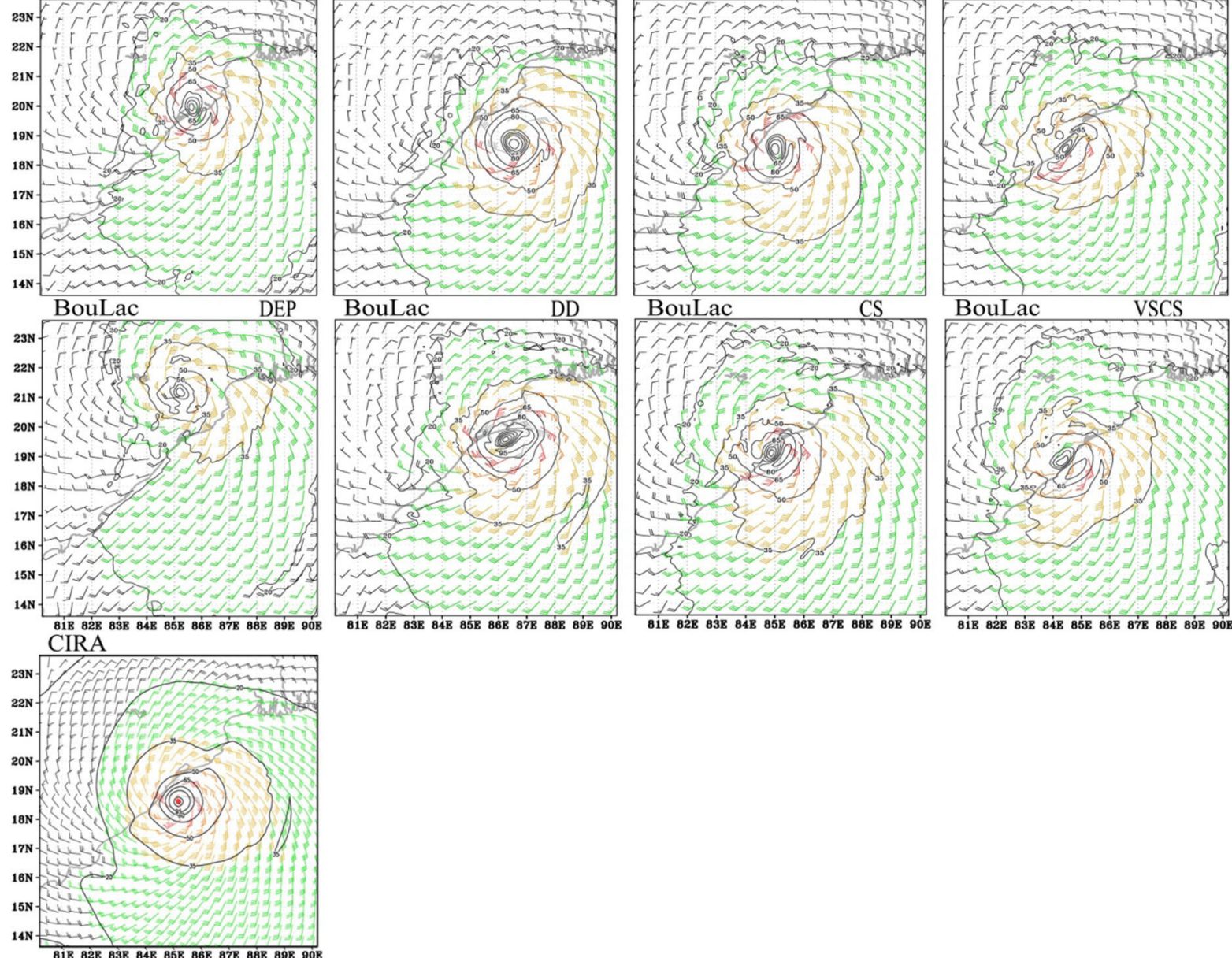

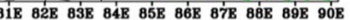

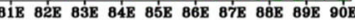

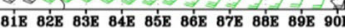

Figure 14

Simulated surface winds at 1200 UTC 12 October 2013 with CIRA multi-satellite observations; the contours indicate the magnitude of the wind in knots

Modelling with all the PBL schemes showed more or less similar rainfall patterns and failed to capture the observed rainfall pattern in the north-western $\mathrm{BOB}$, and the maximum rainfall (approx. $200 \mathrm{~mm} /$ day) over the land portion is well matched with IMD and CPC. The overestimation of rainfall over the north-western $\mathrm{BOB}$ is found to be less $(20 \mathrm{~mm} /$ day) in the simulations with DEP-stage initialization and high (60-80 mm/day) with the advanced stages of model initialization. Particularly,

\begin{tabular}{|l|lll|}
\hline & Journal : Small 24 & Dispatch : 11-10-2018 & Pages : 27 \\
& Article No. : 2017 & $\square$ LE & $\square$ TYPESET \\
MS Code : PAAG-D-18-00007 & $\square \mathrm{CP}$ & $\downarrow$ DISK \\
\hline
\end{tabular}



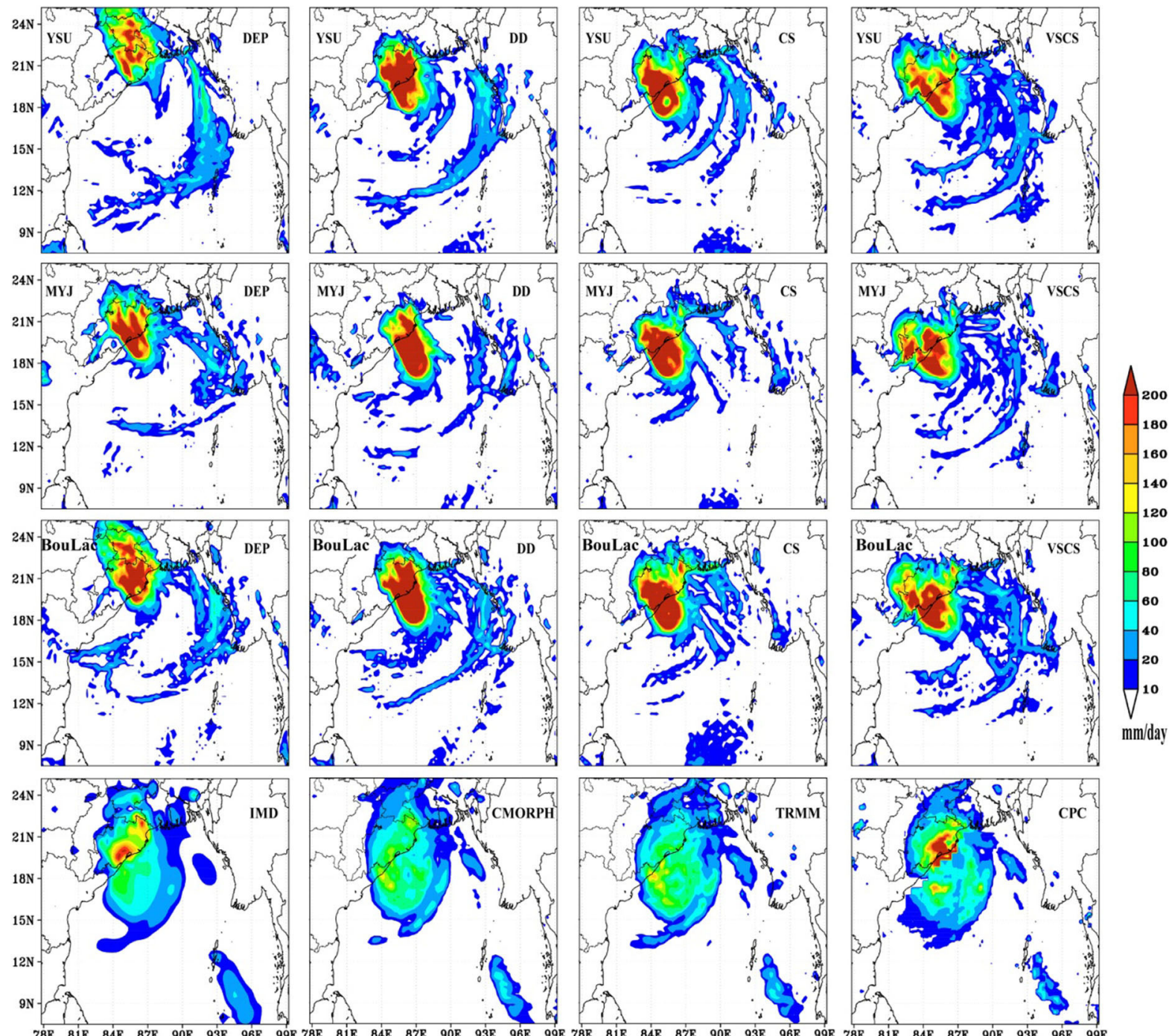

Figure 15

Comparison of simulated 24-h accumulated rainfall for TC Phailin on 12 October 2013 with the observational estimates obtained from IMD, CMORPH, TRMM and CPC

1002 the model in the three PBL experiments simulated 1003 higher rainfall (more than $80 \mathrm{~mm} /$ day) over the left 1004 forward sector of the storm, and considering the 1005 RMSE limits of CMORPH and TRMM (13 mm/day), 1006 the model significantly over-estimated rainfall over 1007 the north-western BOB. The dislocation of rainfall at 1008 the DEP stage of the model initialization is mainly 1009 due to the quick translation of movement in the 1010 simulated storm. The results of the model with the 1011 initializations at DD and CS stages indicate the 1012 observed rainfall over the land region of Orissa and 1013 southern parts of west Bengal are overpredicted. The model initialization at the VSCS stage shows the rainfall pattern over land nearly matches the spatial distribution and amount of rainfall. Though intensity analysis indicates the MYJ scheme produced a weaker-intensity storm with advanced stages of initializations, the relative decrease in simulated rainfall has not been observed. Out of the four model stages of storm initiation, the model initialized at the VSCS stage matches well with the observed rainfall pattern, and the simulated rainfall pattern by the BouLac scheme is close to the observed intensity and
1014 1015 1016 1017 1018 1019 1020 1021 1022 1023 1024

\begin{tabular}{|l|lll|}
\hline & Journal : Small 24 & Dispatch : 11-10-2018 & Pages : 27 \\
& Article No. : 2017 & $\square$ LE & $\square$ TYPESET \\
MS Code : PAAG-D-18-00007 & $\square \mathrm{CP}$ & $\nabla$ DISK \\
\hline
\end{tabular}



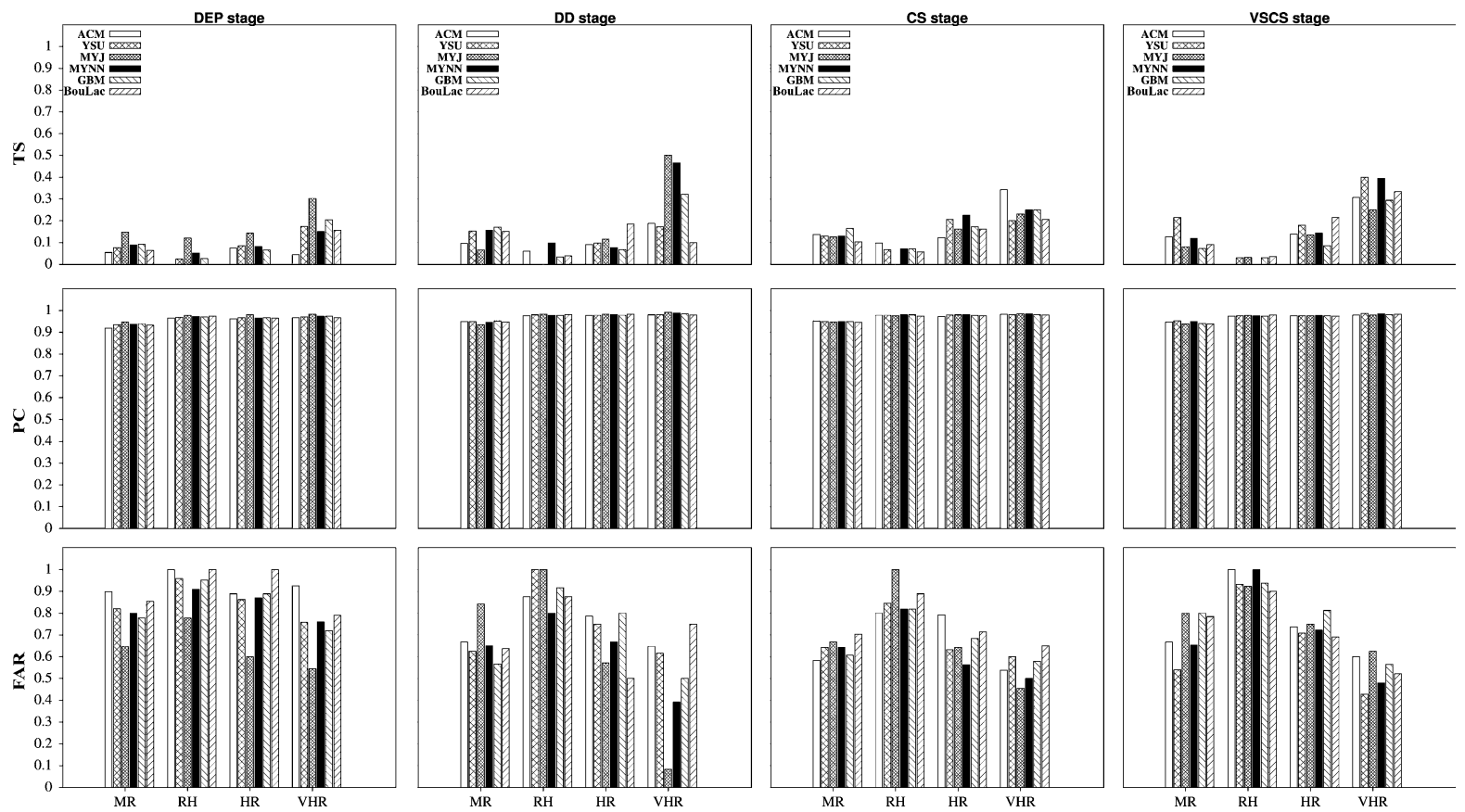

Figure 16

Rainfall error statistics (TS, PC and FAR) computed with IMD gridded rainfall for TC Phailin on 12 October 2013

1025

1026

1027

1028

1029

1030

1031

1032

1033

1034

1035

1036

1037

1038

1039

1040

1041

1042

1043

1044

1045

1046

1047

1048 the symmetric precipitation pattern, followed by the MYJ and YSU schemes.

The PBL schemes predicted rainfall reasonably well over the coastal region, excess rainfall over the ocean region and slight underestimation is also noticed over land regions. To estimate the quantitative skill of the model with different PBL schemes on TC Phailin rainfall, a few skill tests have been conducted and presented in Fig. 16. The skill test was applied at each stage (DEP, DD, CS and VSCS) of the cyclone with different categories of the rainfall like moderate rain $(\geq 10$ to $\leq 35.5 \mathrm{~mm})$, rather heavy (RH; $\geq 35.6$ to $\leq 64.4 \mathrm{~mm}$ ), heavy rain (HR; $\geq 64.5$ to $\leq 124.4 \mathrm{~mm}$ ) and very heavy rain (VHR; $\geq 124.5$ to $\leq 244.4 \mathrm{~mm}$ ). The cyclone induced rainfall over the region, i.e., from $78^{\circ} \mathrm{E}-$ $96^{\circ} \mathrm{E}$, and $9^{\circ} \mathrm{N}-26^{\circ} \mathrm{N}$ is only considered to compute the statistics.

Various thresholds such as TS, PC and FAR have been calculated from Eqs. (1)-(3) and results are presented in Fig. 16. The threat score of all PBL schemes is not up to the mark for all threshold categories, except VHR. This indicates the PBL schemes produced fewer hits and also the model forecasts have grid-to-grid displacement error which implies the presence of a spatial error. However, in all stages, the simulations are good for VHR, and hence it can be concluded that though there are spatial errors in the predicted rainfall and the simulations are good for higher thresholds. Among the selected PBL schemes, the MYJ scheme followed by the MYNN scheme has better TS. The higher PC values at all stages suggest the maximum hit rate, and it is increasing towards the higher threshold. Even though the grid point rainfall predicted well (hit rate is more), the highest false alarm is also noticed and hence the lower values of TS are observed. The higher values of FAR are seen more in the DD stage than other stages and a reduction of FAR can be seen towards the VSCS stage. From the above analysis, the PBL schemes can predict the grid-to-grid rainfall accurately with more non-occurrence rainfall.

1049

1050

1051

1052

1053

1054

1055

1056

1057

1058

1059

1060

1061

1062

1063

1064

1065

1066

\section{Summary and Conclusions}

1067

In this study, the sensitivity of boundary layer 1068 processes initialized at various stages of a TC using

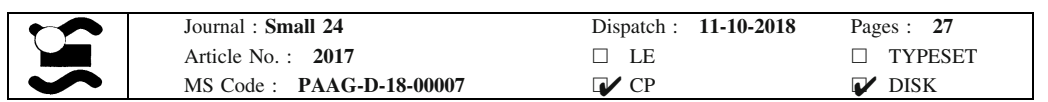


1070 the WRF-ARW model is studied. Six PBL sensitivity 1071 experiments (YSU, ACM, MYNN, BouLac, GBM 1072 and MYJ schemes) are conducted by varying initial 1073 conditions at four different TC stages. A total of 1074 seven post-monsoon cyclones formed over the BOB 1075 are chosen for this analysis. The performance of the 1076 model with different PBL schemes is evaluated 1077 against the best track data from IMD considering the 1078 parameters CSLP, MSW, the RMW and TRMM satellite-derived rainfall estimates. Out of all the six PBL schemes, the MYJ scheme followed by the ACM scheme exhibits higher deviations in simulating TC intensity, while the BouLac scheme followed by the YSU scheme produced minimum errors. The simulations with various PBL schemes show that the first-order non-local PBL schemes (YSU and ACM schemes) predicted the track positions with less error than 1.5 order than the local PBL schemes (MYNN, BouLac, GBM and MYJ schemes) predicted. The track error simulated with PBL schemes is highly sensitive to the initial model conditions. However, it becomes less sensitive with all PBL schemes, as simulated TC parameters are more or less in agreement with observations when the model is initialized at advanced TC stages. All schemes over-estimated the intensity of the TCs during the model initialization at DEP, DD and CS stages; the BouLac scheme produced better intensity compared to observations.

The performance analysis of seven cyclones suggests that TC Phailin was found to be best-simulated TC with least errors followed by TC Hudhudh. So, the model sensitivity to PBL schemes is studied by considering the detailed analysis of TC Phailin. The time series analysis of TC Phailin suggests that the model initialization with a weak cyclonic vortex and local turbulence schemes seems to perform better than non-local, and vice versa when the model is initialized with a well-defined cyclonic vortex. Also, the BouLac scheme produced stronger winds at an observed intensification rate, except with initialization at the DEP stage of the TC stage, while the MYJ scheme produced relatively weaker winds compared to the other four PBL schemes.

The results of radial-height cross-section of TC winds suggest that the strong surface inflow and upper tropospheric outflow is well simulated with the model initializations at advanced stages of the storm. Among the PBL schemes, the BouLac scheme simulated better representation of the structures of gradient and radial winds in the mature stage of the storm, while the MYJ scheme was close to the observed pattern when the model was initialized at the DEP stage; when the error limits of observational estimates (CIRA) are considered, the simulated differences found in PBL schemes, particularly with the BouLac and MYJ schemes, are highly significant. Also, the surface analysis of winds confirms that the MYJ scheme performed better with the DEP stage initialization and the BouLac scheme performed well with initializations at the DD and CS stages.

The rainfall simulated with all the schemes is over-estimated for TC Phailin during landfall. Among the four stages of TC initialization, the VSCS stage matches well with the observed rainfall, especially with the BouLac scheme followed by the MYJ and YSU schemes. The ACM PBL scheme produced more rainfall over the ocean followed by YSU and MYNN. The multi-category analysis of rainfall also confirms the PBL schemes initialized during the mature storm stages seem to produce realistic rainfall distributions.

In this study, model simulations are carried out with horizontal resolution of 27 and $9 \mathrm{~km}$ and with 51 levels. Model sensitivity to the horizontal and vertical resolution is well known from previous studies (Bhaskar Rao et al. 2009); the variation of vertical resolution (Bhaskar Rao et al. 2010) especially impacts the performance of TC predictions with PBL parameterization schemes. Thus, the results of the assessment may vary with the spatial and vertical resolution of the model which is outside of the scope of the selected experimental design.

\section{Acknowledgements}

The authors thank the ISRO-RESPOND project for providing financial support and necessary facilities to carry out the research work. We also thank the UGC, Government of India for providing the high-performance computing cluster (HPC) to carry out the experiments. The Indian Meteorological Department is acknowledged for providing the best track data and

\begin{tabular}{|l|lll|}
\hline & Journal : Small 24 & Dispatch : 11-10-2018 & Pages : 27 \\
& Article No. : 2017 & $\square$ LE & $\square$ TYPESET \\
& MS Code : PAAG-D-18-00007 & $\square \mathrm{CP}$ & $\checkmark$ DISK \\
\hline
\end{tabular}


1161 DWR products. The authors acknowledge the Coop1162 erative Institute for Research in the Atmosphere 1163 (CIRA) and NOAA for providing various real-time 1164 cyclone products and CMORPH rainfall data used for 1165 the study.

\section{6}

\section{REFERENCES}

Anthes, R. A. (1982). Tropical cyclones: Their evolution, structure and effects. Ephrata: American Meteorological Society.

Bhaskar Rao, D. V., \& Hari Prasad, D. (2006). Numerical prediction of the Orissa super-cyclone: Sensitivity to the parameterization of convection, boundary layer and explicit moisture processes. Mausam, 57(1), 61-78.

Bhaskar Rao, D. V., \& Hari Prasad, D. (2007). Sensitivity of tropical cyclone intensification to boundary layer and convective processes. Natural Hazard, 41(3), 429-445.

Bhaskar Rao, D. V., Hari Prasad, D., \& Srinivas, D. (2009). Impact of horizontal resolution and the advantages of the nested domains approach in the prediction of tropical cyclone intensification and movement. Journal of Geophysical Research, 114(D11106), 24.

Bhaskar Rao, D. V., Hari Prasad, D., Srinivas, D., \& Anjaneyulu, Y. (2010). Role of vertical resolution in numerical models towards the intensification, structure and track of tropical cyclones. Marine Geodesy, 33(4), 338-355.

Blackadar, A. K. (1978). Modeling pollutant transfer during daytime convection, In Preprints Fourth Symposium on Atmospheric Turbulence, Diffusion and Air Quality. Reno, Am. Meteor. Soc., pp. 443-447.

Bougeault, P., \& Lacarrere, P. (1989). Parameterization of orography-induced turbulence in a Mesobeta-Scale model. Monthly Weather Review, 117, 1872-1890.

Braun, S. A., \& Tao, W.-K. (2000). Sensitivity of high resolution of hurricane Bob (1991) to planetary boundary layer parameterizations. Monthly Weather Review, 128, 3941-3961.

Chandrasekar, R., \& Balaji, C. (2012). Sensitivity of tropical cyclone Jal simulations to physics parameterizations. Journal of Earth System Science, 121, 923-946.

Chandrasekar, R., \& Balaji, C. (2016). Impact of physics parameterization and 3DVAR data assimilation on prediction of tropical cyclones in the Bay of Bengal region. Natural Hazards, $80(1), 223-247$.

Cohen, A. E., Cavallo, S. M., Coniglio, M. C., \& Brooks, H. E. (2015). A review of planetary boundary layer parameterization schemes and their sensitivity in simulating southeastern U.S. cold season severe weather environments. Weather and Forecasting, 30, 591-612. https://doi.org/10.1175/WAF-D-14-00105.1.

Dasari, H., Rao, V. B., Ramakrishna, S. S. V. S., Paparao, G., \& Ramesh Kumar, P. (2017). On the movement of tropical cyclone LEHAR. Earth Systems and Environment, 1, 21. https://doi.org/ 10.1007/s41748-017-0025-7.

Deshpande, M., Pattnaik, S., \& Salvekar, P. S. (2010). Impact of physical parameterization schemes of numerical simulation of super cyclone Gonu. Natural Hazards, 55, 211-231. https://doi. org/10.1007/s11069-010-9521-x.

Durre, I., Vose, R. S., \& Wuertz, D. B. (2006). Overview of the integrated global radiosonde archive. Journal of Climate, 19(1), 53-68.
Fovell, R. G., \& Su, H. (2007). Impact of cloud microphysics on hurricane track forecasts. Geophysical Research Letters, 34, L24810. https://doi.org/10.1029/2007GL031723.

Gray, W. M. (1968). Global view of the origin of tropical disturbances and storms. Monthly Weather Review, 96, 669-700.

Greeshma, M. M., Srinivas, C. V., Yesubabu, V., Naidu, C. V., Baskaran, R., \& Venkatraman, B. (2015). Impact of local data assimilation on tropical cyclone predictions over the Bay of Bengal using the ARW model. Annales Geophysicae, 33, 805-828.

Grenier, H., \& Bretherton, C. S. (2001). A moist PBL parameterization for large-scale models and its application to subtropical cloud-topped marine boundary layers. Monthly Weather Review, 129, 357-377.

Hari Prasad, K. B. R. R., Srinivas, C. V., Satyanarayana, A. N. V., Naidu, C. V., Baskaran, R., \& Venkatraman, B. (2015). Formulation of stability-dependent empirical relations for turbulent intensities from surface layer turbulence measurements for dispersion parameterization in a lagrangian particle dispersion model. Meteorology and Atmospheric Physics, 127(4), 435-450. https://doi.org/10.1007/s00703-015-0373-5.

Hill, K. A., \& Lackmann, G. L. (2009). Analysis of idealized tropical cyclone simulations using the Weather Research and Forecasting model: Sensitivity to turbulence parameterization and grid spacing. Monthly Weather Review, 137, 745-765.

Holtslag, A. A. M., \& Boville, B. A. (1993). Local versus nonlocal boundary-layer diffusion in a global climate model. Journal of Climate, 6, 1825-1842.

Hong, S. Y., Noh, Y., \& Dudhia, J. (2006). A new vertical diffusion package with an explicit treatment of entrainment processes. Monthly Weather Review, 134, 2318-2341.

Hong, S. Y., \& Pan, H. L. (1996). Nonlocal boundary layer vertical diffusion in a medium-range forecast model. Monthly Weather Review, 124, 2322-2339.

Kanase, R. D., Mukhopadhyay, P., \& Salvekar, P. S. (2015). Understanding the role of cloud and Convective processes in simulating the weaker tropical cyclones over Indian Seas. Pure and Applied Geophysics, 172(6), 1751-1779. https://doi.org/10. 1007/s00024-014-0996-3.

Kanase, R. D., \& Salvekar, P. S. (2015). Effect of physical parameterization schemes on track and intensity of cyclone LAILA using WRF model. Asia-Pacific Journal of Atmospheric Sciences, 51, 205-227. https://doi.org/10.1007/s13143-0150071-8.

Kepert, J. D. (2012). Choosing a boundary layer parameterisation for tropical cyclone modeling. Monthly Weather Review, 140, 1427-1445.

Knaff, J. A., Sampson, C. R., DeMaria, M., Marchok, T. P., Gross, J. M., \& McAdie, C. J. (2007). Statistical tropical cyclone wind radii prediction using climatology and persistence. Weather and Forecasting, 22, 781-791. https://doi.org/10.1175/WAF1026.1.

Kumar, V., Jain, S. K., \& Singh, Y. (2010). Analysis of long-term rainfall trends in India. Hydrological Sciences Journal, 55(4), 484-496. https://doi.org/10.1080/02626667.2010.481373.

Langousis, A., Veneziano, D., \& Chen, S. (2009). Boundary layer model for moving tropical cyclones. In J. Elsner \& T. Jagger (Eds.), Hurricanes and climate change. Boston: Springer.

Ma, Z., Fei, J., Huang, X., \& Cheng, X. (2012). Sensitivity of tropical cyclone intensity and structure to vertical resolution in WRF. Asia-Pacific Journal of Atmospheric Science, 48(1), 67-81. https://doi.org/10.1007/s13143-012-0007-5.

\begin{tabular}{|l|lll|}
\hline & Journal : Small 24 & Dispatch : 11-10-2018 & Pages : 27 \\
Article No. : 2017 & $\square$ LE & $\square$ TYPESET \\
& MS Code : PAAG-D-18-00007 & $\checkmark \mathrm{CP}$ & $\checkmark$ DISK \\
\hline
\end{tabular}


Mandal, M., Mohanty, U. C., \& Raman, S. (2004). A Study on the impact of parameterization of physical processes on prediction of tropical cyclones over the Bay of Bengal with NCAR/PSU mesoscale model. Natural Hazards, 31, 391-414.

Mandal, M., Singh, K. S., Balaji, M., \& Mohapatra, M. (2016). Performance of WRF-ARW model in real-time prediction of Bay of Bengal cyclone 'Phailin'. Pure and Applied Geophysics, 173(5), 1783-1801.

McFarquhar, G. M., Zhang, H., Heymsfield, G., Hood, R., Dudhia, J., Halverson, J. B., et al. (2006). Factors affecting the evolution of Hurricane Erin (2001) and the distributions of Hydrometeors: Role of microphysical processes. Journal of Atmospheric Science, 63, 127-150.

Mellor, G. L., \& Yamada, T. (1982). Development of a turbulence closure model for geophysical fluid problems. Reviews of Geophysics and Space Physics, 20, 851-875.

Mohanty, U. C., Mandal, M., \& Raman, S. (2004). Simulation of Orissa Super Cyclone (1999) using PSU/NCAR mesoscale model. Natural Hazards, 31, 373-390.

Mohanty, U. C., Osuri, K. K., \& Pattanayak, S. (2014). Mesoscale modelling for tropical cyclone forecasting over the North Indian Ocean. Monitoring and prediction of tropical cyclones in the Indian Ocean and Climate Change (pp. 274-286). Dordrecht: Springer.

Mohanty, U. C., Osuri, K. K., Routray, A., Mohapatra, M., \& Pattanayak, S. (2010). Simulation of Bay of Bengal tropical cyclones with WRF model: Impact of initial and boundary conditions. Marine Geodesy, 33(4), 294-314.

Monsoon 2014 A report, IMD Met. Monograph: ESSO Document No.: ESSO/IMD/SYNOPTIC MET/01(2015)/17.

Montgomery, M. T., Smith, R. K., \& Nguyen, S. V. (2010). Sensitivity of tropical-cyclone models to the surface drag coefficient. Quarterly Journal Royal Meteorological Society, 136, 1945-1953.

Murphy, A. H. (1993). What is a good forecast? An essay on the nature of goodness in weather forecasting. Weather and Forecasting, 8, 281-293.

Nakanishi, M., \& Niino, H. (2004). An improved Mellor-Yamada level-3 model with condensation physics: Its design and verification. Boundary-Layer Meteorol., 112, 1-31.

Osuri, K. K., Mohanty, U. C., \& Routray, A. (2014). Role of surface roughness length on simulation of cyclone Aila. Monitoring and prediction of tropical cyclones in the Indian Ocean and climate change (pp. 255-262). Dordrecht: Springer.

Osuri, K. K., Mohanty, U. C., Routray, A., Kulkarni, M. A., \& Mohapatra, M. (2012). Customization of WRF-ARW model with physical parameterization schemes for the simulation of tropical cyclones over North Indian Ocean. Natural Hazards, 63, 1337-1359.

Osuri, K. K., Mohanty, U. C., Routray, A., Mohapatra, M., \& Niyogi, D. (2013). Real-time track prediction of tropical cyclones over the North Indian Ocean using the ARW model. Journal of Applied Meteorology and Climatology, 52, 2476-2492.

Osuri, K. K., Mohanty, U. C., Routray, A., \& Niyogi, D. (2015). Improved prediction of Bay of Bengal tropical cyclones through assimilation of Doppler weather radar observations. Monthly Weather Review, 143, 4533-4560.

Pattnaik, S., \& Krishnamurti, T. N. (2007). Impact of cloud microphysical processes on hurricane intensity, part 1: Control run. Meteorology and Atmospheric Physics, 97, 117-126.
Raghavan, S., \& Sen Sarma, A. K. (2000). Tropical cyclone impacts in India and neighbourhood. In P. Roger \& P. Roger (Eds.), Storms (pp. 339-356). London: Routledge.

Samala, B. K., Nagaraju, C., Banerjee, S., Kaginalkar, A., \& Dalvi, M. (2013). Study of the Indian summer monsoon using WRFROMS regional coupled model simulations. Atmospheric Science Letters, 14, 20-27. https://doi.org/10.1002/as12.409.

Sateesh, M., Srinivas, C. V., \& Raju, P. V. S. (2017). Numerical simulation of tropical cyclone thane: role of boundary layer and surface drag parameterization schemes. Natural Hazards, 89, 1255-1271.

Singh, K. S., \& Bhaskaran, P. K. (2017). Impact of PBL and convection parameterization schemes for prediction of severe land-falling Bay of Bengal cyclones using WRF-ARW model. Journal of Atmospheric and Solar-Terrestrial Physics. https:// doi.org/10.1016/j.jastp.2017.11.004.

Smith, R. K., Montgomery, M. T., \& Nguyen, V. S. (2009). Tropical cyclone spin-up revisited. Quarterly Journal Royal Meteorological Society, 135, 1321-1335.

Smith, R. K., Montgomery, M. T., \& Thomsen, G. L. (2014). Sensitivity of tropical-cyclone models to the surface drag coefficient in different boundary-layer schemes. Quarterly Journal of the Royal Meteorological Society, 140, 792-804.

Smith, R. K., \& Thomsen, G. L. (2010). Dependence of tropicalcyclone intensification on the boundary-layer representation in a numerical model. Quarterly Journal of the Royal Meteorological Society, 136, 1671-1685.

Srikanth, M., Satyanarayana, A. N. V., Srinivas, C. V., \& Tyagi, Bhishma. (2016). Performance evaluation of PBL schemes of ARW model in simulating thermo-dynamical structure of premonsoon convective episodes over Kharagpur using STORM data sets. Pure and Applied Geophysics, 173(5), 1803-1827.

Srinivas, C. V., Bhaskar Rao, D. V., Yesubabu, V., Baskaran, R., \& Venkatraman, B. (2012). Tropical cyclone predictions over the Bay of Bengal using the high-resolution advanced research weather research and forecasting (ARW) model. Quarterly Journal Royal Meteorological Society, 139, 1810-1825.

Srinivas, C. V., Venkatesan, R., Bhaskar Rao, D. V., \& Hariprasad, D. (2007). Numerical simulation of Andhra severe cyclone (2003): Model sensitivity to boundary layer and convection parameterization. Pure and Applied Geophysics, 164, 1-23.

Srinivas, C. V., Yesubabu, V., Hari Prasad, K. B. R. R., \& Venkatraman, B. (2013). Real-time prediction of a severe cyclone 'Jal' over Bay of Bengal using a high-resolution mesoscale model WRF (ARW). Natural Hazards, 65, 331-357.

Sunilkumar, K., Narayana Rao, T., Saikranthi, K., \& Purnachandra Rao, M. (2015). Comprehensive evaluation of multisatellite precipitation estimates over India using gridded rainfall data. Journal of Geophysical Research: Atmospheres, 120, 8987-9005. https://doi.org/10.1002/2015JD023437.

Thomas, Ancy, Samala, B. K., \& Kaginalkar, A. (2014). Simulation of North Indian Ocean tropical cyclones using RAMS numerical weather prediction model. Tropical Cyclone Research and Review, 3(1), 44-52.

W.M.O., Recommendations for the verification and intercomparison of QPFs and PQPFs from Operational NWP Models, 2008; Revision 2, WWRP 2009-1, TD-No.1485, 34PP.

Xie, B., Fung, J. C. H., Chan, A., \& Lau, A. K. H. (2012). Evaluation of nonlocal and local planetary boundary layer schemes in the WRF Model. Journal of Geophysical Research, 117, D12103. https://doi.org/10.1029/2011JD017080.

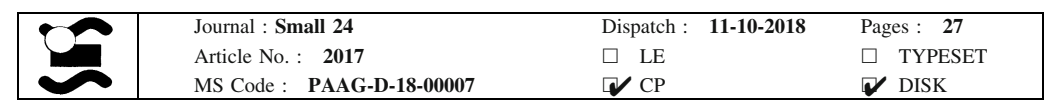


1401 Yesubabu, V., Srinivas, C. V., Hari Prasad, K. B. R. R., Baskaran,

1402 R. (2014a). A study on the impact of observation assimilation

1403 on the numerical simulation of tropical cyclones JAL and

1404 THANE using 3DVAR. https://doi.org/10.1007/s00024-013-

1405 0741-3.
Yesubabu, V., Srinivas, C. V., Ramakrishna, S. S. V. S., \& Hari Prasad, K. B. R. R. (2014b). Impact of period and timescale of FDDA analysis nudging on the numerical simulation of tropical cyclones in the Bay of Bengal. Natural Hazards, 74(3), 2109-2128. https://doi.org/10.1007/s11069-014-1293-2.
1406

1407

1408

1409

1410

1411

1412

(Received January 9, 2018, revised September 22, 2018, accepted October 9, 2018)

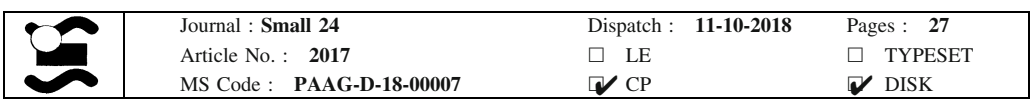




\section{Journal : 24}

Article : 2017

\section{Author Query Form}

\section{Please ensure you fill out your response to the queries raised below and return this form along with your corrections}

Dear Author

During the process of typesetting your article, the following queries have arisen. Please check your typeset proof carefully against the queries listed below and mark the necessary changes either directly on the proof/online grid or in the 'Author's response' area provided below

\begin{tabular}{|l|l|l|}
\hline Query & Details Required & Author's Response \\
\hline AQ1 & Dear Author(s): kindly note the minor edit to the article title. & \\
\hline AQ2 & $\begin{array}{l}\text { Please confirm if the author names are presented accurately and in the correct sequence (given } \\
\text { name, middle name/initial, family name) for [K. Vijaya Kumari, S. Karuna Sagar, D. Hari } \\
\text { Prasad, S. Vijaya Bhaskara Rao] }\end{array}$ & \\
\hline AQ3 & $\begin{array}{l}\text { Bhaskar Rao et al. 2007 has been changed to Bhaskar Rao and Hari Prasad 2007 so that this } \\
\text { citation matches the list. }\end{array}$ & \\
\hline AQ4 & Langousis 2009 has been changed to Langousis et al. 2009 so that this citation matches the list. & \\
\hline AQ5 & $\begin{array}{l}\text { Reference: Reference Bhaskara Rao et al. (2007) was mentioned in the manuscript; however, } \\
\text { this was not included in the reference list. As a rule, all mentioned references should be present } \\
\text { in the reference list. Please provide the reference details to be inserted in the reference list. }\end{array}$ & \\
\hline AQ6 & $\begin{array}{l}\text { References (Bhaskara Rao et al. 2007, Knaff and DeMaria 2006, Demuth et al. 2004) was } \\
\text { provided in the reference list; however, this was not mentioned or cited in the manuscript. As a } \\
\text { rule, if a citation is present in the text, then it should be present in the list. Please provide the } \\
\text { location of where to insert the reference citation in the main body text. }\end{array}$ & \\
\hline AQ7 & $\begin{array}{l}\text { Chandrasekhar et al. (2012) has been changed to Chandrasekar and Balaji (2012) so that this } \\
\text { citation matches the list. }\end{array}$ & \\
\hline AQ8 & $\begin{array}{l}\text { Chandrasekhar et al. 2016 has been changed to Chandrasekar and Balaji 2016 so that this } \\
\text { citation matches the list. }\end{array}$ & \\
\hline AQ9 & $\begin{array}{l}\text { Singh et al. 2017 has been changed to Singh and Bhaskaran 2017 so that this citation matches } \\
\text { the list. }\end{array}$ & \\
\hline AQ10 & $\begin{array}{l}\text { Kindly note the acronyms AMSU and IGRA have not been defined; please define all } \\
\text { acronyms. }\end{array}$ & \\
\hline AQ11 & Kindly note the acronym POD has not been defined; please define. & \\
\hline AQ12 & $\begin{array}{l}\text { Hill et al. 2009 has been changed to Hill and Lackmann 2009 so that this citation matches the } \\
\text { list. }\end{array}$ & \\
\hline AQ13 & Equations 1 to 3 are cited in the text but no equations are given. Kindly check and confirm. & \\
\hline
\end{tabular}

